\title{
Gödel-McKinsey-Tarski and Blok-Esakia for Heyting-Lewis Implication
}

\author{
Jim de Groot ${ }^{1}$, Tadeusz Litak ${ }^{2}$, and Dirk Pattinson ${ }^{1}$ \\ 1 School of Computing The Australian National University \\ jim.degroot@anu.edu.au, dirk.pattinson@anu.edu.au \\ 2 Chair For Theoretical Computer Science FAU Erlangen-Nuremberg \\ tadeusz.litak@fau.de
}

\begin{abstract}
Heyting-Lewis Logic is the extension of intuitionistic propositional logic with a strict implication connective that satisfies the constructive counterparts of axioms for strict implication provable in classical modal logics. Variants of this logic are surprisingly widespread: they appear as Curry-Howard correspondents of (simple type theory extended with) Haskell-style arrows, in preservativity logic of Heyting arithmetic, in the proof theory of guarded (co)recursion, and in the generalization of intuitionistic epistemic logic.

Heyting-Lewis Logic can be interpreted in intuitionistic Kripke frames extended with a binary relation to account for strict implication. We use this semantics to define descriptive frames (generalisations of Esakia spaces), and establish a categorical duality between the algebraic interpretation and the frame semantics. We then adapt a transformation by Wolter and Zakharyaschev to translate Heyting-Lewis Logic to classical modal logic with two unary operators. This allows us to prove a Blok-Esakia theorem that we then use to obtain both known and new canonicity and correspondence theorems, and the finite model property and decidability for a large family of Heyting-Lewis logics.
\end{abstract}

\section{Introduction}

Modern modal logic was invented by C. I. Lewis $[63,66,68]$ as the theory of strict implication 3 . Lewis assumed a classical propositional base and definability of -3 in terms of unary modal operators ${ }^{1}$ as $\square(\varphi \rightarrow \psi)$. Consequently, while implication-like connectives are intensively studied in other areas (relevance, substructural, counterfactual and conditional logics), some of which in fact are of modal origin [76, Ch. 6], modal logic in a narrow sense gradually came to focus mostly on unary boxes and diamonds, with Lewis' original strict implication -3 falling into disuse.

Recently, Litak and Visser [71] investigated -3 over an intuitionistic rather than classical propositional base, using intuitionistic Kripke frames with an additional binary relation interpret strict implication. While a $\square$-modality can be obtained from -3 via $\square \varphi:=\top-3 \varphi$, strict implication is not definable from $\square$. The constructive implication -3 was first studied in the context of preservativity for theories over Heyting Arithmetic HA (§ 2.5). Arrows in functional programming [48] yield another important class of Heyting-Lewis implications via the CurryHoward correspondence (§ 2.3). Yet another nontrivial variant of -3 arises when one generalizes

\footnotetext{
${ }^{1}$ Curiously, Lewis was not using $\square$ as a primitive, so in fact his intuitionistically problematic definition of $\varphi \dashv \psi$ was $\neg \diamond(\varphi \wedge \neg \psi)$. See [71, App. D] for an account of problems caused by Lewis' use of a Boolean propositional base, namely trivialization $[64,67]$ of his original system $[63,66]$, which in turn finally lead him to propose systems S1-S3 [68, App. 2] as successive "lines of retreat" [88]. Lewis considered S4 and S5, suggested by Becker [8], too strong to provide a proper account of strict implication [68, p. 502] and appeared frustrated with later development of modal logic $[71, \S 2.1]$. Yet, despite his supportive attitude towards non-classical logics, he seems to have mentioned Brouwer only once (favourably) [65], and does not appear to have ever referred to, or even be familiar with subsequent work of Kolmogorov, Heyting or Glivenko [71, § 2.2].
} 
Artemov and Protopopescu's [5] approach to intuitionistic epistemic logic (§ 2.4). Even where 3 is reducible to $\square$ in terms of theoremhood, it can still be a more useful primitive. This has been argued in the proof theory of guarded (co)recursion (Exm. 2.4 in $\S 2.2$; see also [71, § 7.2]).

While Kripke semantics has obvious benefits, it does not provide a fully global completeness theorem for arbitrary extensions of the minimal Heyting-Lewis system iA (§ 2.1), even in the limitative modal or superintuitionistic cases. In contrast, the systems presented here are amenable to algebraic semantics, called Heyting-Lewis algebras. These are obtained by fusing Heyting algebras with so-called weak Heyting algebras [22] over the shared lattice reduct (§ 3.1). To combine advantages of algebraic and relational semantics, one typically works with dual representations of algebras called descriptive frames. These can sometimes be viewed as topological spaces, analogous to Esakia spaces for intuitionistic propositional logic. Like the algebraic semantics, they give completeness, but are often easier to manipulate and transform, e.g., to prove the finite model property and decidability. We provide suitable Heyting-Lewis dualities in $\S 3$.

Intuitionistic logics with natural Kripke semantics can often be viewed as fragments of classical modal logics determined by the same Kripke structures. In particular, formulae of IPC can be identified via the Gödel-McKinsey-Tarski translation with those formulae of modal logic S4 where every subformula is prefixed with $\square$. Extensions of S4 satisfying the Grzegorczyk axiom Grz of conversely well-founded posets correspond to extensions of IPC: the Blok-Esakia theorem $[17,37,38]$ establishes an isomorphism between the lattice of intermediate logics and the lattice of S4Grz-logics.

Wolter and Zakharyaschev [108,109] extended the Gödel-McKinsey-Tarski translation to provide a Blok-Esakia-style isomorphism between intuitionistic unimodal logics and classical bimodal logics. This proved a fruitful approach, enabling the use of well-developed classical metatheory in proofs of correspondence, completeness, canonicity, the finite model property, and decidability results. In $\S 4$, we generalise their Blok-Esakia result to the Heyting-Lewis setting.

In order to put this transfer apparatus to good use, in $\S 5$ we set out to prove the finite model property and decidability for classical bimodal logics. In particular, we prove this for (cofinal) transitive subframe logics. These results can then be transferred to a large class of HeytingLewis logics (with an additional axiom enforcing that the relation interpreting -3 is transitive). In both $\S 4$ and $\S 5$, care and ingenuity is required with Wolter and Zakharyaschev's original techniques: some results transfer smoothly to the more general setting, while other lemmas and theorems require subtler proofs, like Prop. 4.12 and Lem. 4.18 and 4.19 below. We are not aware of similar transfer results between an intuitionistic source language involving binary modalities and a classical language involving exclusively unary ones.

Earlier version. This is the technical report corresponding to the similarly named paper accepted for publication at LICS 2021.

\section{Syntax, Axioms and Examples}

Define the language $\mathcal{L}_{-3}$ by the grammar

$$
\varphi::=p|\top| \perp|\varphi \wedge \varphi| \varphi \vee \varphi|\varphi \rightarrow \varphi| \varphi-\jmath \varphi
$$

where $p$ ranges over some fixed set of propositional atoms At. As usual, $\neg \varphi:=\varphi \rightarrow \perp$. Furthermore, $\square \varphi:=\top \neg \varphi$. The unary connectives $\neg$ and $\square$ bind strongest, next comes $\rightarrow$, then $\wedge$ and $\vee$, and lastly $\rightarrow$. 


\subsection{Axioms and Rules for Arrows}

We define Heyting-Lewis Logic (the system iA following [71] $)^{2}$ as the extension of the intuitionistic propositional calculus (IPC) with the axioms

$$
\begin{aligned}
& \mathrm{K}_{\mathrm{a}}((\varphi-3 \psi) \wedge(\varphi-3 \chi)) \rightarrow(\varphi-3(\psi \wedge \chi)) \\
& \operatorname{Di}((\varphi-\jmath \chi) \wedge(\psi \jmath \chi)) \rightarrow((\varphi \vee \psi) \dashv \chi) \\
& \operatorname{Tr}((\varphi-3 \psi) \wedge(\psi-3 \chi)) \rightarrow(\varphi-\jmath \chi)
\end{aligned}
$$

and the arrow necessitation rule:

$$
\mathrm{N}_{\mathrm{a}} \frac{\varphi \rightarrow \psi}{\varphi-3 \psi}
$$

We also call iA the (base) Heyting-Lewis logic. The system obtained by removing Di from the above axiomatization will be denoted as $\mathrm{i}^{-}$. In several important applications below, one needs to distinguish between the $\mathrm{iA}^{-}$- and $\mathrm{iA}$-variants. Only the latter can be given a sound and complete Kripke-style semantics (see $\S 3.2$ ), and the results established in this paper generally require the presence of Di (cf. $\S 6$ ).

A (iA-) logic is a set of $\mathcal{L}_{-3}$-formulae containing all of the above axioms, and closed under $\mathrm{N}_{a}$ and uniform substitiution. Given $\Lambda, \Gamma \subseteq \mathcal{L}_{-}, \Lambda \oplus \Gamma$ denotes the smallest logic containing $\Lambda \cup \Gamma$. We write $\Lambda \oplus\{\varphi\}$ as $\Lambda \oplus \varphi$ and in the special case of $\Lambda=\mathrm{iA}\left(\Lambda=\mathrm{iA}^{-}\right)$, we write $\mathrm{i}-\varphi\left(\mathrm{i}-\varphi^{-}\right)$.

\subsection{Intuitionistic Normal Modal Logics (with Box)}

One easily shows $[52,53,71]$ that the defined box is normal: the axiom $\mathrm{K}_{\square}$ and the rule $\mathrm{N}_{\square}$ obtained by substituting $T$ for $\varphi$ in $\mathrm{K}_{\mathrm{a}}$ and $\mathrm{N}_{\mathrm{a}}$, respectively, are derivable in $i \mathrm{~A}^{-}$, just like

$$
\square(\varphi \rightarrow \psi) \rightarrow \varphi-3 \psi
$$

Thus, postulating as an axiom the opposite implication

$$
\operatorname{Box}(\varphi-3 \psi) \rightarrow \square(\varphi \rightarrow \psi)
$$

not only makes 3 interdefinable with $\square$ (and makes Di derivable even over iA ${ }^{-}$[71, Lem 4.4c]), but reduces the study of Heyting-Lewis logics extending i-Box to the study of normal modal extensions of Int $\mathrm{K}_{\square}$, i.e., extensions of IPC closed under $\mathrm{K}_{\square}, \mathrm{N}_{\square}$ and uniform substitutions in the language $\mathcal{L}_{\square}$ (replacing $\varphi-3 \psi$ with $\square(\varphi \rightarrow \psi)$ ).

Conversely, this means that the Heyting-Lewis Logic of strict implication subsumes a large class of intuitionistic modal logics. In this rather broad area $[21,28,33,57,72,87,96,110]^{3}$ one sometimes includes a separate $\diamond$ connective undefinable in i-Box, but there are often good reasons to work in the setting of a single $\square$ added to the IPC signature, particularly when studying the Curry-Howard-Lambek correspondence for a specific functor/type operator. Examples of interest include:

2.1 Example. The (monoidal) comonadic box of constructive S4 [2,14], obtained by extending IntK $\mathrm{K}_{\square}$ with

\footnotetext{
${ }^{2}$ Litak and Visser [71] use the name "Lewis arrow" for -3 , which leads to names such as iA, or to the use of a as a subscript.

${ }^{3}$ Sotirov [98] claims accurately that "there is an outburst of interest in this topic each decade" since Fitch's 1948 paper [40].
} 


$$
\mathrm{T} \varphi \rightarrow \square \varphi \quad 4_{\square} \square \varphi \rightarrow \square \square \varphi
$$

is used to control staged computation $[29,86]$.

2.2 Example. The strength axiom ${ }^{4}$ :

$\mathrm{S}_{\square} \varphi \rightarrow \square \varphi$.

yields IntK $\mathrm{K}_{\square} \oplus \mathrm{S}_{\square}{ }^{5}$, the (inhabitation) logic of Haskell's applicative functors (idioms) [77], as noted in recent references $[73,92]$ (cf. $\S 2.3)$. It has also been proposed as a minimal system of intuitionistic epistemic logic (cf. § 2.4).

2.3 Example. Extending IntK $\mathrm{K}_{\square} \oplus \mathrm{S}_{\square}$ with

$$
\mathrm{C} 4 \square \square \varphi \rightarrow \square \varphi
$$

yields the Propositional Lax Logic PLL [28,39,46]. This is known as the Curry-Howard correspondent of strong monads [9,58,82], but has numerous other application in hardware verification [39], access control [43], epistemic logic [5], or topos logic [46].

2.4 Example. The strong Löb axiom

$\mathrm{SL}_{\square}(\square \varphi \rightarrow \varphi) \rightarrow \varphi$

entails $\mathrm{S}_{\square}[81 \text {, Lem. } 3.2]^{6}$ and hence is equivalent to

$\mathrm{SL}_{\square}^{\prime}(\square \varphi \rightarrow \varphi) \rightarrow \square \varphi$.

Furthermore, the system $\operatorname{lntK}_{\square} \oplus \mathrm{SL}_{\square}$ is easily seen to be equivalent to the one obtained by extending $\operatorname{IntK}_{\square} \oplus \mathrm{S}_{\square}$ with the (ordinary) Löb axiom

$$
\mathrm{L}_{\square} \square(\square \varphi \rightarrow \varphi) \rightarrow \square \varphi
$$

$\mathrm{SL}_{\square}$ has been studied in the context of extensions of Heyting Arithmetic with the completeness principle [103] (see § 2.5). However, computer scientists may recognize it as an axiom for the modality for guarded (co)recursion [84,85], also known as the later operator $[10,15,54,56]$, next clock tick [59,60] or guardedness type constructor [7]. Proof systems developed in this context often treat (definable) 3 as a primitive connective [1,26,84]. Thus effectively they are proof systems for $\mathrm{i}-\mathrm{SL}_{\square} \oplus$ Box rather than for $\mathrm{IntK}_{\square} \oplus \mathrm{SL}_{\square}$; see $[71, \S 7.2]$ for a detailed discussion.

\subsection{Haskell Arrows (with Choice)}

Over $\mathrm{iA}^{-}$, the strength axiom $\mathrm{S}_{\square}$ is equivalent to

$$
\mathrm{S}_{\mathrm{a}}(\varphi \rightarrow \psi) \rightarrow(\varphi-3 \psi)[71, \text { Lem 4.10]. }
$$

From a type-theoretic perspective, the axioms of $\mathrm{i}_{-} \mathrm{S}_{\mathrm{a}}{ }^{-}$correspond to inhabitation laws of Hughes arrows [48], where an arrow is a binary type constructor that represents computations, here given in Haskell notation:

\footnotetext{
${ }^{4}$ Strength of the functor interpreting $\square$ in a categorical semantics of modal proofs [2, 14, 34,73, 92] corresponds to the validity of $(\varphi \wedge \square \psi) \rightarrow \square(\varphi \wedge \psi)$, but this is derivable from $\mathrm{S}_{\square}$ when $\square$ is normal [73, Sec. 6]. Classically, i- $S_{\square}$ collapses to a fairly non-interesting system [73, Rem. 25].

${ }^{5}$ We are abusing the $\oplus$ notation for logics in $\mathcal{L}_{\square}$, replacing closure under axioms and rules of iA with closure under axioms and rules of IntK $_{\square}$.

${ }^{6}$ This is a "deboxed" version of well-known derivation of transitivity from the standard Löb axiom [20, Thm. 18]. The above reference provides a categorical translation of this derivation.
} 


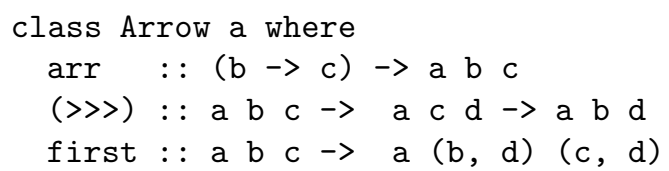

Reading strict implication $b-3 c$ as the type of arrows with domain $b$ and codomain $c$, the first function, arr stipulates that every function of type $\mathrm{b} \rightarrow \mathrm{c}$ can be interpreted as a computation from $\mathrm{b}$ to $\mathrm{c}$, which is precisely $\mathrm{S}_{\mathrm{a}}$. The second function allows us to compose arrows, which is modally captured by Tr. Finally, the direct modal transliteration of first and $\mathrm{K}_{\mathrm{a}}$ are interderivable over IPC [71, Lem. 4.1]. This leaves the Di axiom that corresponds to a frequently used extension of arrows, the so-called arrows with choice [49]. This amounts to stipulating an extra operation

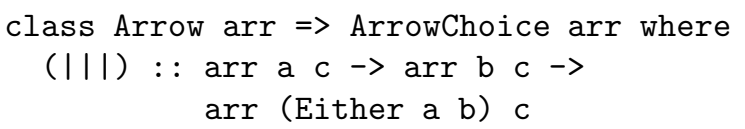

One can set up the same correspondences that are usually exhibited between modal logic and type theory in a (strong) monad setting, e.g., give a realisability interpretation [58] of HeytingLewis proofs as functions in a type theory with a chosen notion of arrow, a propositions-astypes interpretation [9], or a categorical semantics [2]. Related work has indeed been done for arrows $[6,55,69,70]$, but inasmuch as we are aware, so far avoiding explicit mention of logic (or Di/Choice). ${ }^{7}$

2.5 Example (Arrow-collapsing Choice). Clearly, the trivial example of arrows, i.e. function spaces, are arrows with choice. The above-mentioned applicative functors are another limiting case: they correspond to arrows with delay [70, Def. 5.1], an operation which simply makes (the type corresponding to) Box inhabited [70]. Finally, monads are equivalent to higher-order arrows $[70, \S 6]$ or arrows with apply $[49, \S 5.2]$, where the apply operation inhabits a type corresponding to one of Lewis' original axioms [68][71, Rem. 7.3]:

$\operatorname{App}_{a}(\varphi \wedge(\varphi-\beta))-3 \psi$.

The logic PLAA $:=\mathrm{i}-\mathrm{S}_{\mathrm{a}} \oplus \mathrm{App}_{\mathrm{a}}$ allows for a decomposition of $\varphi-3 \psi$ as $\varphi \rightarrow \square \psi$ [70] [71, Lem 4.17f]. This also entails derivability of $\mathrm{Di}$ [71, Lem 4.17g], which in the Haskell context was already noted in Hughes' original paper [49, §5.2]. ${ }^{8}$

2.6 Example (Nontrivial Choice). An example that does not trvialise -3 is provided by Kleisli arrows: given a monad $M$, we define the type of Kleisli arrows over types $a$ and $b$ as arrows in the Kleisli category given by $M$, that is, A $a b=a \rightarrow M b$. Just like with function spaces, this allows us to define $f\|\| g=[f, g]$ as the co-pair. For co-Kleisli arrows, i.e., defining A $a b=M a \rightarrow b$, we need to additionally require that the monad $M$ comes equipped with a distributive law over coproducts, viz. $M(a+b) \rightarrow M a+M b$. Finally, list processors are presented as arrows with choice in [49], where for the choice operation |||, the interleaving pattern in the output is modelled on the interleaving of the input.

\footnotetext{
${ }^{7}$ To the best of our knowledge, the only explicit (if brief) discussion of the Curry-Howard connection between Haskell arrows and $\mathrm{i}_{\mathrm{a}}{ }^{-}$is found in Litak and Visser [71, § 7.1].

${ }^{8}$ The logical perspective seems to cast a light on the controversy whether arrows are "stronger" than applicative functors $[70,77]$. Putting aside the general question of whether one takes as a measure of strength the capability to inhabit more types or rather to allow more distinctions, in the presence of -3 , the situation is not as clear-cut as in the unary case, where (monadic) PLL simply extends (applicative) IntK $\square \oplus S_{\square}$. Defining arrows over the latter set of axioms via delay yields $\mathrm{i}-\mathrm{S}_{\mathrm{a}}{ }^{-} \oplus$ Box, whereas inhabiting apply with Appa yields PLAA. These are two incomparable systems.
} 
2.7 Example (Arrows without Choice). An example of arrows that do not come equipped with choice are automata that transform elements of type $a$ to elements of type $b$ that satisfy the isomorphism $\mathrm{A} a b \cong a \rightarrow b \times(\mathrm{A} a b)$. Another non-example are functions on infinite streams: given two functions Stream $a \rightarrow \operatorname{Stream} b$ and Stream $b \rightarrow$ Stream $c$, there is no generic way to construct a function Stream $(a+b) \rightarrow$ Stream $c$.

\subsection{Intuitionistic Epistemic Logic of Entailment}

From an (intuitionistic) epistemic logical point of view, Heyting-Lewis logic can be used to reason about entailment, interpreting " $\varphi-3 \psi$ " as "the agent knows that $\varphi$ entails $\psi$ ". This allows us to not only reason about the knowledge of an agent, but also about their deductive abilities. We recover statements about agent's knowledge via $\square \psi=\top\lrcorner \psi$.

This idea leads to a generalisation of Artemov and Protopopescu's intuitionistic epistemic logic IEL [5]. We briefly discuss generalizations of two basic principles they postulate: coreflection and intuitionistic reflection.

An intuitionistic implication holds only if there exists a proof for it. As a consequence of this proof, our Heyting-Lewis agent $[5, \S 2.1]$ knows the implication:

\section{Intuitionistic implication $\Rightarrow$ knowledge of implication}

Syntactically, this simply means validity of the strength axiom $S_{a}$ (or equivalently $S_{\square}$ ), i.e., coreflection $\varphi \rightarrow \square \varphi[5]$.

Conversely, known implications cannot be false. Therefore one cannot intuitionistically falsify any implication that is known. This gives rise to the following generalisation of intuitionistic reflection $(\square \varphi \rightarrow \neg \neg \varphi)$

IR $(\varphi-3 \psi) \rightarrow \neg \neg(\varphi \rightarrow \psi)$.

One could say that knowledge of the entailment of $\psi$ from $\varphi$ prevents one from proving that $\neg \psi$ given $\varphi$, i.e.,

$\operatorname{IR}^{\prime}(\varphi-\psi) \rightarrow(\varphi \rightarrow \neg \neg \psi)$

Since IPC $\vdash \neg \neg(\varphi \rightarrow \psi) \leftrightarrow(\varphi \rightarrow \neg \neg \psi)$, both axioms are equivalent.

2.8 Definition. The intuitionistic epistemic logic of entailment is given by IELE $=\mathrm{iA} \oplus \mathrm{S}_{\mathrm{a}} \oplus \mathrm{IR}$.

There is no natural way to Box-collapse IELE. Knowledge of an entailment $\varphi-\beta \psi$ does not imply the existence of an intuitionistic proof, or knowledge thereof.

Besides, as a consequence of the strength axiom we have $(\varphi-3 \psi) \rightarrow(\varphi \rightarrow \square \psi)$ (see [71, Lem. $4.10 \mathrm{~b}]$ ). Its converse, however, need not be true. While $\varphi$ may imply knowledge of $\psi$, there is no reason it should entail intuitionistic truth of $\psi$. Thus we do not wish to have the Hughes law collapsing arrows in the monadic setting (§ 2.3), i.e., we do not stipulate

Hug $(\varphi \rightarrow \square \psi) \rightarrow(\varphi-3 \psi)$.

We will see in Prop. 3.10 below that Box, Hug and the converse of IR are not derivable from IELE. Nevertheless, knowledge of entailment and intuitionistic implication are not entirely unrelated. Generalising [5, Thm. 3.5(3)], we find:

2.9 Proposition. In IELE we have $\neg(\varphi-3 \psi) \leftrightarrow \neg(\varphi \rightarrow \psi)$.

Proof. The direction from left to right follows from $\left(\mathrm{S}_{\mathrm{a}}\right)$. It follows from (IR) that $\neg \neg \neg(\varphi \rightarrow$ $\psi) \rightarrow \neg(\varphi-3 \psi)$ and since $\neg(\varphi \rightarrow \psi) \rightarrow \neg \neg \neg(\varphi \rightarrow \psi)$ this proves the converse.

Investigation of further meaningful epistemic axioms to add to IELE is future research. See $\S 6$ for a discussion. 


\subsection{Arithmetical Interpretations}

There is more than one possible interpretation of -3 in theories over Heyting Arithmetic (HA) or other base systems such as Intuitionistic Elementary Arithmetic (IEA). A framework of schematic logics accounts for this variety of interpretations $[71, \S 5],[74, \S 4]$, broadly generalizing the well-known provability interpretation of the classical Löb logic [20]. The most important arithmetical interpretation is that of $\Sigma_{1}$-preservativity, historically the first context in which constructive -3 appeared $[52,53,100,101,103]$. This interpretation makes inclusion of not only $\mathrm{S}_{\square}$, but even $\mathrm{Di}$ in the base system problematic.

More broadly, one can define $\Delta$-preservativity for a theory $T$, where $\Delta$ is an elementary class of arithmetical sentences containing $T$. First, define $A-{ }_{\Delta, T} B$ as the following relation: for all $S \in \Delta$, if $T \vdash S \rightarrow A$, then $T \vdash S \rightarrow B$. For each $\Delta$ and (a fixed axiomatization of) $T$, this yields a binary arithmetical predicate encoding the corresponding relation on Gödel numbers in the language of IEA. ${ }^{9}$ Now given a mapping $f$ from At to arithmetical sentences, extend it inductively to the whole $\mathcal{L}_{-3}$, using the Gödel encoding and the predicate in question to interpret -3 . It is immediate to see that such an interpretation makes $\mathrm{iA}^{-}$valid for every $T$, $\Delta$ and $f$. In order to ensure the validity of Di in the logic of $\Sigma_{1}$-preservativity of a given $T$, one needs additional conditions such as $T$ 's $T$-provable closure under q-realizability, which does hold for HA or Markov Arithmetic MA [71, § 5.4.1], but not in all of their extensions [74, Ex 5.6].

Unary $\square$ encodes arithmetical provability under this interpretation, but while the usual Löb axiom $L_{\square}$ from $\S 2.2$ remains in the provability logic of $\mathrm{HA}$, the said logic contains many principles failing in the provability logic of Peano Arithmetic (PA) [71, § 5.3]. In fact, unlike the classical case [97], a complete axiomatization of the intuitionistic $\mathcal{L}_{\square}$-provability logics remains elusive $[4,50,51,100,102]$ and one of the main motivations for studying the $\mathcal{L}_{-3}$-logic of $\Sigma_{1}$-preservativity has been that this seemingly more challenging task may yield more natural axiomatic principles.

Furthermore, in the setting of constructive -3 , just the addition of $L_{\square}$ is not sufficient to derive the famous Explicit Fixpoint Theorem of provability logic [11, 19, 20, 93, 99, 104] and one can consider several mutually incomparable axioms which restore it [74]. One of them is

$$
\mathrm{P}(\varphi \jmath \psi) \rightarrow \square(\varphi-3 \psi)
$$

which ensures exactly the transitivity of the modal relation interpreting -3 [74, Th 10.1] and plays a central rôle in the finite model property and decidability results in $\S 5$ below.

Finally, inasmuch as the strength axiom $S_{\square}\left(\right.$ or $S_{a}$ ) is concerned, it is obviously not valid in preservativity or provability logic of either HA or PA. Nevertheless, one can consider the system $\mathrm{HA}^{*}$ which incorporates $\mathrm{S}_{\square}$ as the so-called completeness principle $[3,31,103][71, \S 5.4 .4]$.

\section{$3 \quad$ Semantics and Duality}

The logics introduced above correspond to varieties of Heyting algebras with binary operators. In particular, the algebraic semantics of $\mathrm{iA}$ is given by Heyting-Lewis algebras, defined in $\S 3.1$ below. After defining these, we recall the relational semantics of iA (§3.2), equip these with collections of admissible subsets to obtain general and descriptive frames $(\S 3.3)$ and prove a categorical duality between the descriptive frames and Heyting-Lewis algebras (§ 3.4).

\subsection{Heyting-Lewis Algebras}

The algebraic semantics of iA looks as follows:

\footnotetext{
${ }^{9}$ One could go further and give a 4 -argument predicate in the language of second-order arithmetic, parametric in both $\Delta$ and the axiomatization of $T$.
} 
3.1 Definition. A Heyting-Lewis algebra or HL-algebra is a tuple of the form

$$
\mathcal{A}:=(A, \top, \perp, \wedge, \vee, \rightarrow, \Im),
$$

where $(A, \top, \perp, \wedge, \vee, \rightarrow)$ is a Heyting algebra and $(A, \top, \perp, \wedge, \vee, \neg)$ is a so-called weak Heyting algebra [22], i.e., $-3: A \times A \rightarrow A$ is a binary operator that satisfies:

$$
\begin{aligned}
& \text { C1 }(a-b) \wedge(a-3 c)=a \dashv(b \wedge c) \\
& \text { C2 }(a-c) \wedge(b-3 c)=(a \vee b)-3 c \\
& \text { C3 }(a-b) \wedge(b-3 c) \leq a-3 c \\
& \text { C4 } a-3 a=\top
\end{aligned}
$$

When no confusion is likely we will write $(A,-3)$ and understand $A$ to be (the set underlying) a Heyting algebra.

An HL-algebra morphism from $(A,-3)$ to $\left(A^{\prime}, \Im^{\prime}\right)$ is a Heyting homomorphism $h: A \rightarrow A^{\prime}$ that additionally satisfies $h(a-3 b)=h(a)-3^{\prime} h(b)$ for all $a, b \in A$. We write HLAs for the category of $\mathrm{HL}$-algebras and $\mathrm{HL}$-algebra morphisms.

The collection of $\mathcal{L}_{-3}$-formulae modulo provable equivalence yields an HL-algebra (the LindenbaumTarski algebra), cf. [74, § 3.1]. In fact, completeness of iA and its extensions can be shown by standard techniques for algebraizable logics [18,41,42,91]. This is not only true for theoremhood, but also for theories induced by the global consequence relation. As this is routine, we skip the details.

\subsection{Strict Implication Frames and Models}

We recall the relational semantics for $\mathcal{L}_{-3}[51, \S 3.4 .2]$, [71, Definition 3.3]. These are intuitionistic Kripke frames (i.e., posets) with an additional binary relation that is used to interpret the strict implication 3 .

3.2 Definition. A strict implication frame, or -3 -frame for short, is a tuple $(X, \preceq, \sqsubset)$ consisting of a poset $(X, \preceq)$ and a binary relation $\sqsubset$ on $X$ that satisfies for all $x, y, z \in X$,

$$
\text { if } \quad x \preceq y \sqsubset z \text { then } x \sqsubset z \text {. }
$$

A - -morphism from $(X, \preceq, \sqsubset)$ to $\left(X^{\prime}, \preceq^{\prime}, \sqsubset^{\prime}\right)$ is a function $f: X \rightarrow X^{\prime}$ that is bounded with respect to both relations. That is, for $R \in\{\preceq, \sqsubset\}$ and all $x, y \in X$ and $z^{\prime} \in X^{\prime}$ :

P1 If $x R y$ then $f(x) R^{\prime} f(y)$;

P2 If $f(x) R^{\prime} z^{\prime}$ then $\exists z \in X$ s.t. $x R z$ and $f(z)=z^{\prime}$;

We write -3 -Frm for the category of -3 -frames and -morphisms.

For a poset $(X, \preceq)$, let $u p(X, \preceq)=\{a \subseteq X \mid$ if $x \in a$ and $x \preceq y$ then $y \in a\}$ be the collection of upsets of $X$. Recall that $u p(X, \preceq)$ can be given a Heyting algebra structure where top, bottom, meet and join are given by $X, \emptyset$, intersection and union, respectively. Implication is defined by

$$
a \rightrightarrows b=\{x \in X \mid \text { if } x \preceq y \text { and } y \in a \text { then } y \in b\} .
$$

Likewise, $\rightarrow$-frames give rise to $\mathrm{HL}$-algebras: 
3.3 Lemma. Every -3 -frame $\mathfrak{X}=(X, \preceq, \sqsubset)$ gives rise to an $\mathrm{HL}$-algebra $\mathfrak{X}^{+}=(u p(X, \preceq), \underline{3})$, where $\underline{3}$ is defined by

$$
a \underline{3} b=\{x \in X \mid \text { if } x \sqsubset y \text { and } y \in a \text { then } y \in b\} .
$$

Proof. If $a$ and $b$ are upsets, then by $(-3-p)$ so is $a \underline{-} \underline{b} b$, so $\underline{-3}$ is well defined. To prove that ( $u p(X, \preceq), \underline{-3})$ is an $\mathrm{HL}$-algebra we need to show that it satisfies C1 to C4 from Definition 3.1. Each of these follows from a straightforward verification.

The algebra $\mathfrak{X}^{+}$is known as the complex algebra of $\mathfrak{X}$.

3.4 Proposition. The assignment $(\cdot)^{+}$extends to a contravariant functor $(\cdot)^{+}:-3-\mathrm{Frm} \rightarrow \mathrm{HLAs}$ by setting $f^{+}=f^{-1}$ for every -3-frame morphism $f$.

Proof. We have already seen that $(\cdot)^{+}$is well defined on objects. If $f:(X, \preceq, \sqsubset) \rightarrow\left(X^{\prime}, \preceq^{\prime}, \sqsubset^{\prime}\right)$ is a -3 -frame morphism, then in particular it is a bounded morphism from $(X, \preceq)$ to $\left(X^{\prime}, \preceq^{\prime}\right)$ and hence $f^{-1}: u p\left(X^{\prime}, \preceq^{\prime}\right) \rightarrow u p(X, \preceq)$ is a Heyting homomorphism. Boundedness of $f$ with respect to $\sqsubset$ entails that $f^{-1}\left(a^{\prime} \underline{\underline{z}}^{\prime} b^{\prime}\right)=f^{-1}\left(a^{\prime}\right) \underline{\underline{3}} f^{-1}(b)$. Functoriality is straightforward.

Thus we can choose upsets of $(X, \preceq)$ as the intepretants of a - -frame $(X, \preceq, \sqsubset)$ and define a - -model as follows.

3.5 Definition. A valuation for a -3 -frame $(X, \preceq, \sqsubset)$ is a function $V$ : At $\rightarrow u p(X, \preceq)$ that assigns to each propositional variable $p$ an upset of $(X, \preceq)$. A - -model is a tuple $(\mathfrak{X}, V)$ of a -3-frame $\mathfrak{X}=(X, \preceq, \sqsubset)$ and a valuation $V$ for $\mathfrak{X}$.

The set of states of a model $\mathfrak{M}=(\mathfrak{X}, V)$ satisfying an $\mathcal{L}_{-3}$-formula $\varphi$ is called the truth set of $\varphi$. It is denoted by $\llbracket \varphi \rrbracket^{\mathfrak{M}}$ and defined recursively by $\llbracket p \rrbracket^{\mathfrak{M}}=V(p)$, $\llbracket \top \rrbracket^{\mathfrak{M}}=X$, $\llbracket \perp \rrbracket^{\mathfrak{M}}=\emptyset$, and

$$
\begin{aligned}
\llbracket \varphi \wedge \psi \rrbracket^{\mathfrak{M}} & =\llbracket \varphi \rrbracket^{\mathfrak{M}} \cap \llbracket \psi \rrbracket^{\mathfrak{M}} & \llbracket \varphi \rightarrow \psi \rrbracket^{\mathfrak{M}} & =\llbracket \varphi \rrbracket^{\mathfrak{M}} \rightrightarrows \llbracket \psi \rrbracket^{\mathfrak{M}} \\
\llbracket \varphi \vee \psi \rrbracket^{\mathfrak{M}} & =\llbracket \varphi \rrbracket^{\mathfrak{M}} \cup \llbracket \psi \rrbracket^{\mathfrak{M}} & \llbracket \varphi-3 \psi \rrbracket^{\mathfrak{M}} & =\llbracket \varphi \rrbracket^{\mathfrak{M}} \underline{-} \llbracket \llbracket \rrbracket^{\mathfrak{M}}
\end{aligned}
$$

If $x \in \llbracket \varphi \rrbracket^{\mathfrak{M}}$ we say that $x$ satisfies $\varphi$, and write $\mathfrak{M}, x \Vdash \varphi$. A model $\mathfrak{M}$ satisfies $\varphi$ if $\llbracket \varphi \rrbracket^{\mathfrak{M}}=X$, notation: $\mathfrak{M} \Vdash \varphi$. A frame $\mathfrak{X}$ is said to satisfy $\varphi$ if every model based on it satisfies $\varphi$, in which case we write $\mathfrak{X} \Vdash \varphi$. If $\Gamma$ is a set of $\mathcal{L}_{-3}$-formulae then we write $\mathfrak{X} \Vdash \Gamma$ if $\mathfrak{X} \Vdash \varphi$ for all $\varphi \in \Gamma$.

The class of -3 -models can be extended to a category with the following notion of morphism.

3.6 Definition. A -3-model morphism from $(\mathfrak{X}, V)$ to $\left(\mathfrak{X}^{\prime}, V^{\prime}\right)$ is a - -frame morphism $f: \mathfrak{X} \rightarrow$ $\mathfrak{X}^{\prime}$ that additionally satisfies $V=f^{-1} \circ V^{\prime}$. We write 3 -Mod for the category of -3-models and their morphisms.

A routine induction on the structure of $\varphi$ shows that:

3.7 Proposition. Let $f: \mathfrak{M} \rightarrow \mathfrak{M}^{\prime}$ be a-3-model morphism. Then for all $x \in X$ and $\varphi \in \mathcal{L}_{-}$ we have

$$
\mathfrak{M}, x \Vdash \varphi \quad \text { iff } \quad \mathfrak{M}^{\prime}, f(x) \Vdash \varphi .
$$

3.8 Corollary. Let $f: \mathfrak{X} \rightarrow \mathfrak{X}^{\prime}$ be a surjective -3 -frame morphism. Then $\mathfrak{X} \Vdash \varphi$ implies $\mathfrak{X}^{\prime} \Vdash \varphi$.

Proof. If $V^{\prime}$ is any valuation for $\mathfrak{X}^{\prime}$, then $V:=f^{-1} \circ V^{\prime}$ is a valuation for $\mathfrak{X}$ such that $f$ : $(\mathfrak{X}, V) \rightarrow\left(\mathfrak{X}^{\prime}, V^{\prime}\right)$ is a -3 -model morphism. It then follows from Prop. 3.7 that $\left(\mathfrak{X}^{\prime}, V^{\prime}\right) \Vdash \varphi$ because $(\mathfrak{X}, V) \Vdash \varphi$ and $f$ is surjective. 


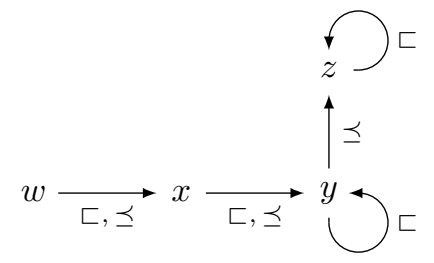

Figure 1: The frame from Prop. 3.10.

It is known (see e.g. [71, Thm. 6.4a] and references therein) that 3 -frames form a sound and complete semantics for iA. We now prove correspondence results for the axioms of IELE and use this to give a sound and complete semantics for IELE. As an example application, we substantiate the claim that IELE does not satisfy Box, Hug, and the converse of IR.

3.9 Proposition. Let $\mathfrak{X}=(X, \preceq, \sqsubset)$ be a 3 -frame.

1) $\mathfrak{X}$ satisfies $\mathrm{S}_{\mathrm{a}}$ iff $x \sqsubset y$ implies $x \preceq y$ for all $x, y \in X$.

2) $\mathfrak{X}$ satisfies IR iff for all $x \in X$ there exists $y \in X$ such that $x \preceq y$ and $x \sqsubset y$.

Proof. Item 1) follows from Lem. 4.10 and Thm. 6.4(c) in [71].

For the second item, suppose there exists $x \in X$ such that no $\sqsubset$-successor of $x$ is also a $\preceq$-successor. Then we can set $V(p)=\uparrow \preceq x$ and $V(q)=\emptyset$, and an easy verification shows that $x \Vdash \varphi \neg \psi$ while $x \Vdash \varphi \rightarrow \neg \neg \psi$. Conversely, let $\mathfrak{X}$ be such that every $x \in X$ has a $\sqsubset$-successor that is also a $\preceq$-successor. Suppose $x \Vdash \varphi-3 \psi$. We aim to prove that $x$ satisfies $\varphi \rightarrow \neg \neg \psi$. So let $y$ be such that $x \preceq y$ and $y \Vdash \varphi$. Then in order to prove that $y \Vdash \neg \neg \psi$, we need to show that $y \preceq z$ implies $z \forall \psi \rightarrow \perp$. By assumption there exists $w \in X$ such that $z \sqsubset w$ and $z \preceq w$. Since $x \preceq z$ and $x \Vdash \varphi \dashv \psi$ we must have $z \Vdash \varphi \neg \psi$. Similarly, as $y \preceq w$ and $y \Vdash \varphi$ we find $w \Vdash \varphi$, and because $z \sqsubset w$ this means $w \Vdash \psi$. Therefore $z \Vdash \psi \rightarrow \perp$. This proves that $y \Vdash \neg \neg \psi$ and hence $x \Vdash(\varphi \rightarrow \neg \neg \psi)$, as desired.

In the presence of the strength axiom, validity of IR is guaranteed by the requirement that every $x \in X$ has a $\sqsubset$-successor. Therefore a - -frame $(X, \preceq, \sqsubset)$ is a frame for IELE if and only if $x \sqsubset y$ implies $x \preceq y$ and every state has a $\sqsubset$-successor. We note that these are the same frames as used for the intuitionistic epistemic logic IEL, cf. [5, Def. 4.2].

3.10 Proposition. The axioms Box, Hug, and the converse of IR are not derivable from IELE.

Proof. Consider the frame $\mathfrak{X}=(X, \preceq, \sqsubset)$ where $X=\{w, x, y, z\}, \sqsubset=\{(w, x),(x, y),(y, y),(z, z)\}$, and $\preceq$ is given by the reflexive and transitive closure of $w \preceq x \preceq y \preceq z$, see Fig. 1. This is a frame for IELE because the relation $\sqsubset$ is contained in $\preceq$, and every state has a $\sqsubset$-successor. It can easily be verified that this does not satisfy the frame conditions corresponding to Box and Hug given in [71, Fig. 6.2], so that $\mathfrak{X} \forall$ Box and $\mathfrak{X} \forall$ Hug. It follows that Box and Hug are not derivable in IELE.

Finally, we show that over $\mathfrak{X},(\varphi \rightarrow \neg \neg \psi)$ does not imply $(\varphi-3 \psi)$. Consider the valuation given by $V(p)=X$ and $V(q)=\{z\}$. Then by construction $x \forall p-3 q$. On the other hand, it follows from $z \Vdash q$ that $t \Vdash \neg q$ for all states $t \in X$, so that $t \Vdash \neg \neg q$ for all $t \in X$. Therefore $x \Vdash p \rightarrow \neg \neg q$, and hence $x$ itself witnesses $x \Vdash(p \rightarrow \neg \neg q) \rightarrow(p-\neg q)$. 


\subsection{General and Descriptive Frames}

Next we define general -3 -frames. These will then be used to obtain a duality result for HLAs (Thm. 3.22). Moreover, we will make extensive use of general frames in $\S 4$ below, where we embed $\mathcal{L}_{3}$ into bimodal classical logic.

3.11 Definition. A general -3 -frame is a tuple $(X, \preceq, \sqsubset, P)$ such that $(X, \preceq, \sqsubset)$ is a -3 -frame and $P \subseteq u p(X, \preceq)$ is a collection of upsets containing $X$ and $\emptyset$, and closed under $\cap, \cup, \rightrightarrows$ and $\underline{3}$. It is called descriptive if additionally it is

- Compact: For every $A \subseteq P$ and $B \subseteq\{X \backslash a \mid a \in P\}$, if $A \cup B$ has the f.i.p. then $\bigcap(A \cup B) \neq \emptyset$;

- $\preceq$-Refined: For all $x, y \in X$, if $x \npreceq y$ then there exists $a \in P$ such that $x \in a$ and $y \notin a$;

- $\sqsubset$-Refined: For all $x, y \in X$, if $x \not \subset y$ then there exist $a, b \in P$ such that $x \in a \underline{3} b$ and $y \in a$ and $y \notin b$.

If $\mathfrak{G}=(X, \preceq, \sqsubset, P)$ is a geneneral $\neg$-frame, we write $\kappa \mathfrak{G}$ for the underlying - -frame $(X, \preceq, \sqsubset)$.

Observe that the reduct $(X, \preceq, P)$ of a general - -frame $\mathfrak{G}=(X, \preceq, \sqsubset, P)$ is a general intuitionistic Kripke frame. Therefore the set $P$ of admissibles forms a sub-Heyting algebra of $u p(X, \preceq)$. Moreover, if $\mathfrak{G}$ is descriptive then $(X, \preceq, P)$ is a descriptive intuitionistic Kripke frame (see e.g. $[23, \S 8.4]$ ). Therefore we may alternatively define a descriptive -3 -frame as a tuple $(X, \preceq, \sqsubset, P)$ such that (i) $(X, \preceq, P)$ is a descriptive intuitionistic Kripke frame, (ii) $P$ is closed under $\underline{-}$, and (iii) $\sqsubset$-refinedness is satisfied.

Since $P$ is closed under $\underline{-}$, we can view $(P, \underline{\underline{3}})$ as a sub-algebra of $(\kappa \mathfrak{G})^{+}$(cf. Lem. 3.3). In particular this implies that $(P, \underline{-3})$ is an $\mathrm{HL}$-algebra, and we denote it by $\mathfrak{G}^{*}=(P, \underline{-3})$.

We now define morphisms between general -3 -frames.

3.12 Definition. A general 3 -frame morphism between $(X, \preceq, \sqsubset, P)$ and $\left(X^{\prime}, \preceq^{\prime}, \sqsubset^{\prime}, P^{\prime}\right)$ is a -3-frame morphism $f:(X, \preceq, \sqsubset) \rightarrow\left(X^{\prime}, \preceq^{\prime}, \sqsubset^{\prime}\right)$ with $f^{-1}\left(a^{\prime}\right) \in P$ for all $a^{\prime} \in P^{\prime}$. Let G-Frm be the category of general 3 -frames and morphisms and D-Frm its full subcategory of descriptive -3-frames.

3.13 Remark. It is well known that descriptive intuitionistic Kripke frames can be viewed as topological spaces with an extra relation, called Esakia spaces [36,38]. As descriptive -3-frames are based on descriptive intuitionistic Kripke frames, this adapts accordingly. Define a strict implication space to be a tuple $(X, \preceq, \sqsubset, \tau)$ such that $(X, \preceq, \tau)$ is an Esakia space and $\sqsubset$ a binary relation on $X$ such that

- $x \preceq y \sqsubset z$ implies $x \sqsubset z$ for all $x, y, z \in X$;

- $\downarrow_{\sqsubset} a=\{x \in X \mid x \sqsubset y$ for some $y \in a\}$ is clopen for every clopen $a \subseteq X$;

- $\uparrow_{\sqsubset} x=\{y \in X \mid x \sqsubset y\}$ is closed in $(X, \tau)$ for all $x \in X$.

These constitute the category SIS, whose morphisms are continuous morphisms that are bounded with respect to both relations. An easy verification shows that

$$
\mathrm{D}-\mathrm{Frm} \cong \mathrm{SIS} .
$$

For details, see Appendix A.1. 
If $f: \mathfrak{G} \rightarrow \mathfrak{G}^{\prime}$ is a general 3 -frame morphisms, then we define $f^{*}:=f^{-1}:\left(\mathfrak{G}^{\prime}\right)^{*} \rightarrow \mathfrak{G}^{*}$. This assignment is well defined by the definition of a general 3 -frame morphism, and it is an $\mathrm{HL}$-algebra morphism as a consequence of Prop. 3.4. We have:

3.14 Proposition. The assignment $(\cdot)^{*}:$ G-Frm $\rightarrow$ HLAs defines a contravariant functor.

A general $ヶ$-frame $\mathfrak{G}=(X, \preceq, \sqsubset, P)$ can be turned into a general $\_$-model by endowing it with an admissible valuation, that is, a map $V:$ At $\rightarrow P$. The interpretation of $\mathcal{L}_{-3}$-formulae in $(\mathfrak{G}, V)$ is defined as in the underlying -3 -model $(\kappa \mathfrak{G}, V)$. We write $\mathfrak{G} \Vdash \varphi$ if $\varphi$ is satisfied in every general -3 -model based on $\mathfrak{G}$. If $\Gamma$ is a set of $\mathcal{L}_{-3}$-formula then we define $\mathfrak{G} \Vdash \Gamma$ as expected. Similar to Cor. 3.8 one can prove:

3.15 Proposition. Let $f: \mathfrak{G} \rightarrow \mathfrak{G}^{\prime}$ be a surjective general frame morphism. Then $\mathfrak{G} \Vdash \varphi$ implies $\mathfrak{G}^{\prime} \Vdash \varphi$.

Since valuations for $\mathfrak{G}$ are in particular valuations for the underlying -3 -frame $\kappa \mathfrak{G}$, validity of a formula $\varphi$ in $\kappa \mathfrak{G}$ implies validity of $\varphi$ in $\mathfrak{G}$. The converse, however, need not be true. If the converse holds for the class of descriptive 3 -frames, then we call $\varphi$ canonical. Similarly, for logics:

3.16 Definition. A logic $\Lambda$ is called canonical if $\mathfrak{G} \Vdash \Lambda$ implies $\kappa \mathfrak{G} \Vdash \Lambda$ for all descriptive ॐ-frames $\mathfrak{G}$.

This notion of canonicity is sometimes called d-persistence, as is the case in $[108,109]$.

3.17 Example. An example of a canonical iA-logic is $i A \oplus \mathrm{S}_{\mathrm{a}}$. To see this, note that by Prop. 3.9(1)) it suffices to prove that for every descriptive frame $\mathfrak{G}=(X, \preceq, \sqsubset, P)$ satisfying $\mathrm{S}_{\mathrm{a}}$ we have: if $x \sqsubset y$ then $x \preceq y$ for all $x, y \in X$. This, in turn, follows from an application of 乃-refinedness.

In $\S \S 4$ and 5 below we will see how canonicity for iA-logics follows from canonicity of classical bimodal logics.

\subsection{Duality}

Recall that every Heyting algebra $A$ gives rise to a descriptive intuitionistic Kripke frame $(p f A, \subseteq, \widetilde{A})$. Here pf $A$ denotes the set of prime filters of $A$, and $\widetilde{A}=\{\widetilde{a} \mid a \in A\}$, where $\widetilde{a}=$ $\{\mathfrak{p} \in p f A \mid a \in \mathfrak{p}\}$. (For details see e.g. [23, §8.2] or [12, §§ 2.2 and 2.3].) We generalise this to construct a general 3 -frame from an $\mathrm{HL}$-algebra. This extends to a functor $(\cdot)_{*}:$ HLAs $\rightarrow$ G-Frm, which is shown to give rise to a duality HLAs ${ }^{\text {op }}$ D-Frm in Thm. 3.22 below.

This duality can also be obtained from [22, Thm. 4.15] by restricting to the right-hand side to WH-spaces [22, Def. 4.4] whose underlying Priestly space is an Esakia space.

3.18 Definition. Let $\mathcal{A}=(A,-3)$ be an $\mathrm{HL}$-algebra and let $(p f A, \subseteq, \widetilde{A})$ be the descriptive intuitionistic Kripke frame dual to $A$. Then we define the general 3 -frame $\mathcal{A}_{*}$ dual to $\mathcal{A}$ to be $\mathcal{A}_{*}=(p f A, \subseteq, \sqsubset, \widetilde{A})$, where

$$
\mathfrak{p} \sqsubset \mathfrak{q} \quad \text { iff } \quad \forall a, b \in A(a-3 b \in \mathfrak{p} \text { and } a \in \mathfrak{q} \text { implies } b \in \mathfrak{q}) .
$$

While it is fairly easy to verify that $\subseteq$ and $\sqsubset$ satisfy the required coherence condition $(-3-\mathrm{p})$, so that ( $p f A, \subseteq, \sqsubset$ ) is a -3 -frame, it is not clear whether $\widetilde{A}$ gives it a general frame structure. In particular, it is not obvious why $\widetilde{A}$ should be closed under $\underline{3}$. In order to prove that it is, we make use of the fact that $\widetilde{\sim}: A \rightarrow \widetilde{A}$ defines an isomorphism of Heyting algebras [23, $\S 8.4]$. In Lem. 3.19 we prove that that it is a iA-morphism, so that consequently $\widetilde{a} \underline{-3} \widetilde{b}=\widetilde{a-3 b} \in \widetilde{A}$. 
3.19 Lemma. Let $\mathcal{A}=(A,-3)$ be an $\mathrm{HL}$-algebra, and let $\mathcal{A}_{*}=(p f A, \subseteq, \sqsubset, \widetilde{A})$ be defined as in Definition 3.18. Then for all $a, b \in A$ we have

$$
\widetilde{a-3 b}=\widetilde{a} \underline{3} \widetilde{b} .
$$

Proof. A proof can be found in Appendix A.2.

As stated, this shows that $\mathcal{A}_{*}$ is a general -3 -frame. In fact:

3.20 Proposition. If $\mathcal{A}=(A,-3)$ is a $\mathrm{HL}$-algebra, then $\mathcal{A}_{*}$ is a descriptive -3-frame.

As a consequence, the map $\widetilde{(\cdot)}: \mathcal{A} \rightarrow\left(\mathcal{A}_{*}\right)^{*}$ is an isomorphism in HLAs. Indeed, it is an isomorphism between the underlying Heyting algebras, so by Lem. 3.19 a bijective HL-algebra morphism, hence an isomorphism because HLAs is a variety of algebras.

Every iA-morphism $h: \mathcal{A} \rightarrow \mathcal{A}^{\prime}$ is in particular a Heyting homomorphism, wherefore $p f h=h^{-1}:\left(p f A^{\prime}, \subseteq, \widetilde{A}^{\prime}\right) \rightarrow(p f A, \subseteq, \widetilde{A})$ is a general intuitionistic Kripke frame morphism. It follows from the definition of $\sqsubset$ that $h^{-1}$ is also a -3 -frame morphism between the underlying -3-frames, so that setting $h_{*}=h^{-1}$ yields a functor $(\cdot)_{*}:$ HLAs $\rightarrow$ G-Frm.

3.21 Lemma. The iso $\widetilde{(\cdot)}: \mathcal{A} \rightarrow\left(\mathcal{A}_{*}\right)^{*}$ is natural in $\mathcal{A}$.

Since the functor $(\cdot)_{*}$ lands in D-Frm, we may view it as a functor HLAs $\rightarrow$ D-Frm. In the converse direction we shall be sloppy and write $(\cdot)^{*}$ for the restriction of $(\cdot)^{*}:$ G-Frm $\rightarrow$ HLAs to D-Frm. Then we get:

3.22 Theorem. The functors $(\cdot)^{*}:$ D-Frm $\rightarrow$ HLAs and $(\cdot)_{*}:$ HLAs $\rightarrow$ D-Frm define a dual equivalence HLAs $\equiv{ }^{\mathrm{op}}$ D-Frm.

As a consequence of the duality of Thm. 3.22 we have:

3.23 Theorem. Every iA-logic is characterised by a class of descriptive -3-frames.

\section{Embedding into Bimodal Classical Logic}

The Gödel-McKinsey-Tarski translation embeds intuitionistic logic into the modal classical logic S4 by prefixing every subformula of an intuitionistic formula with $\square[45,78]$. This was extended to the class of all intermediate logics by Dummett and Lemmon [35]. The structure of the lattice of intermediate logics was later investigated by Maksimova and Rybakov [75], Blok [17] and Esakia [37,38]. Most notably, this led to what is now known as the Blok-Esakia theorem, which classifies all modal companions of an intermediate logic and, as a corollary, establishes an isomorphism between the lattice of intermediate logics and the lattice of S4logics that satisfy the Grzegorczyk axiom. Fischer Servi and Shehtman further generalised it to embed intuitionistic logic with a normal unary modality into the bimodal classical logic S4 $\otimes K[94,95]$. This was then exploited by Wolter and Zakharyaschev [108, 109] to transfer results on completeness, decidability, the finite model property, and tabularity between modal intuitionistic logic and bimodal classical logic.

In this section we generalise the Gödel-McKinsey-Tarski translation to an embedding $t$ of $\mathcal{L}_{-3}$ into a bimodal classical language. We briefly recall some facts, fix notation for bimodal classical logic and define our syntactic translation in $\S 4.1$. Thereafter, in $\S 4.2$, we define translations from general -3 -frames to general S4K-frames and vice versa, and examine their properties. In $\S 4.3$ we define and investigate modal companions of iA-logics. This all sets us up to prove an analogue of the Blok-Esakia theorem for iA-logics in $\S 4.4$. As a consequence we obtain an isomorpism between the lattice of iA-logics and a certain sublattice of $(\mathrm{S} 4 \otimes \mathrm{K})$-logics. Moreover, it allows us to prove preservation properties between iA-logics and their modal companions. 


\subsection{General S4K-frames}

We denote the fusion of S4 (with modality $\square_{i}$ ) and $K$ (with modality $\square_{m}$ ) by S4K. The subscript $\mathrm{i}$ indicates that $\square_{i}$ arises from embedding intuitionistic logic into S4. The box with subscript $\mathrm{m}$ is an additional modality used for the translation of -3 .

4.1 Definition. An S4K-frame is a tuple $\left(X, R_{\mathrm{i}}, R_{\mathrm{m}}\right)$ consisting of a set $X$, and a pre-order $R_{\mathrm{i}}$ and a binary relation $R_{\mathrm{m}}$ on $X$. A p-morphism from $\left(X, R_{\mathrm{i}}, R_{\mathrm{m}}\right)$ to $\left(X^{\prime}, R_{\mathrm{i}}^{\prime}, R_{\mathrm{m}}^{\prime}\right)$ is a function $f: X \rightarrow X^{\prime}$ that is bounded with respect to both relations. An S4K-frame $\left(X, R_{\mathrm{i}}, R_{\mathrm{m}}\right)$ is called a bimodal Heyting-Lewis frame or S4BHL-frame if it satisfies

$$
R_{\mathrm{i}} \circ R_{\mathrm{m}} \subseteq R_{\mathrm{m}}
$$

We write S4K and S4BHL for the categories of S4K-frames and S4BHL-frames, respectively, and p-morphisms.

Observe that every -3-frame can be conceived of as an S4BHL-frame. However, the latter are still slightly more general than -3 -frames because $R_{\mathrm{i}}$ is only required to be a pre-order, rather than a partial order. An easy verification shows that satisfaction of (1) is equivalent to validity of

BHL $\square_{\mathrm{m}} \varphi \rightarrow \square_{\mathrm{i}} \square_{\mathrm{m}} \varphi$

Furthermore, standard Sahlqvist-style results entail that the normal bimodal logic S4BHL obtained by extending S4K with BHL is in fact (strongly) complete with respect to S4BHL frames.

We now recall the definition of general S4K-frames, for a textbook reference see e.g. $[16, \S 5]$ or $[23, \S 8.1]$.

4.2 Definition. A general S4K-frame is a tuple $\left(X, R_{\mathrm{i}}, R_{\mathrm{m}}, P\right)$ that consists of an S4K-frame $\left(X, R_{\mathrm{i}}, R_{\mathrm{m}}\right)$ and a Boolean subalgebra $P \subseteq \mathcal{P} X$ of the powerset Boolean algebra of $X$, such that $P$ is closed under

$$
\begin{aligned}
{[\mathrm{i}]: \mathcal{P} X \rightarrow \mathcal{P} X: a } & \mapsto\left\{x \in X \mid x R_{\mathrm{i}} y \text { implies } y \in a\right\} \\
{[\mathrm{m}]: \mathcal{P} X \rightarrow \mathcal{P} X: a } & \mapsto\left\{x \in X \mid x R_{\mathrm{m}} y \text { implies } y \in a\right\}
\end{aligned}
$$

A general S4K-frame morphism $f:\left(X, R_{\mathrm{i}}, R_{\mathrm{m}}, P\right) \rightarrow\left(X^{\prime}, R_{\mathrm{i}}^{\prime}, R_{\mathrm{m}}^{\prime}, P^{\prime}\right)$ is a p-morphism between the underlying S4K-frame such that $f^{-1}\left(a^{\prime}\right) \in P$ whenever $a^{\prime} \in P^{\prime}$. We write G-S4K for the category of general S4K-frames and morphisms.

General S4BHL-frames, descriptive S4K-frames, and descriptive S4BHL-frames are defined as usual (see e.g. [16, Def. 5.59 and 5.65]), and their categories are denoted by G-S4BHL, D-S4K and D-S4BHL, respectively.

Finally, we write $\mathrm{Grz}_{\mathrm{i}}$ and $\mathrm{Grz}_{\mathrm{m}}$ for the Grzegorczyk axiom

$$
\operatorname{Grz} \square(\square(p \rightarrow \square p) \rightarrow p) \rightarrow p
$$

written with $\square_{i}$ (resp. $\square_{m}$ ) in place of $\square$. This axiom plays an important rôle in the original Blok-Esakia theorem, and also appears in our adaptation of it.

Now we are ready to define (two versions of) the GMT translation. 
4.3 Definition (The GMT translation for $\mathcal{L}_{-3}$ ). Let $\mathcal{L}_{\mathrm{i}, \mathrm{m}}$ be the language of classical bimodal logic with modalities $\square_{i}$ and $\square_{m}$, and define the translation $t$ of $\mathcal{L}_{3}$ into $\mathcal{L}_{i, m}$ recursively by

$$
\begin{aligned}
t(p)=\square_{\mathbf{i}} p & \quad t(\top)=\top \quad t(\perp)=\perp \\
t(\varphi \wedge \psi) & =\square_{\mathbf{i}}(t(\varphi) \wedge t(\psi)) \\
t(\varphi \vee \psi) & =\square_{\mathbf{i}}(t(\varphi) \vee t(\psi)) \\
t(\varphi \rightarrow \psi) & =\square_{\mathbf{i}}(t(\varphi) \rightarrow t(\psi)) \\
t(\varphi \rightarrow \psi) & =\square_{\mathbf{i}} \square_{\mathrm{m}}(t(\varphi) \rightarrow t(\psi))
\end{aligned}
$$

Note that the translation of $\square \varphi=\top-3 \varphi$ is given by $t(\square \varphi)=\square_{i} \square_{m}(t(T) \rightarrow t(\varphi))$, which is S4K-equivalent to $\square_{i} \square_{\mathrm{m}} t(\varphi)$, so $t$ extends the translation used by Wolter and Zakharyaschev in [109]. Furthermore, observe that over S4BHL, the -3 clause can be replaced by $\square_{\mathbf{m}}(t(\varphi) \rightarrow t(\psi))$, a fact that we will use tacitly in what follows.

\subsection{Translations of Frames}

Next, we define functors $\widehat{\sigma}:$ G-Frm $\rightarrow$ G-S4K and $\widehat{\rho}:$ G-S4K $\rightarrow$ G-Frm. We prove that the composition $\widehat{\rho} \widehat{\sigma}$ is naturally isomorphic to the identity, and that both $\widehat{\sigma}$ and $\widehat{\rho}$ preserve descriptiveness. The transformations $\widehat{\rho}$ and $\widehat{\sigma}$ are based on the maps $\rho$ and $\sigma$ that translate between general intuitionistic Kripke frames and general S4-frames [23, $\S 3.9$ and 8.3]. We add a hat to distinguish them from the maps $\rho$ and $\sigma$ that translate between iA-logics and S4K-logics defined in $\S$ 4.3. A similar construction was carried out in [108,109], and we point out the differences when we encounter them.

We have already seen that every -3-frame can be conceived of as an S4K-frame. This extends to general frames:

4.4 Definition. Given a general - -frame $\mathfrak{G}=(X, \preceq, \sqsubset, P)$, let $\widehat{\sigma} \mathfrak{G}$ be the general S4K-frame

$$
\widehat{\sigma} \mathfrak{G}=(X, \preceq, \sqsubset, \widehat{\sigma} P),
$$

where $\widehat{\sigma} P$ is the Boolean closure of $P$ in $\mathcal{P} X$ (the powerset of $X$ ). For a general 3 -frame morphism $f$, let $\widehat{\sigma} f=f$.

4.5 Lemma. If $\mathfrak{G}$ is a general -3-frame, then $\widehat{\sigma} \mathfrak{G}$ is a general S4BHL-frame. Moreover, for any $a \in \widehat{\sigma} P$ we have $[\mathrm{m}] a \in P$.

Proof. We need to show that $\widehat{\sigma} P$ is closed under [i] and [m]. The former follows from [23, Lem. 8.32 and 8.33]. For the latter (and the "moreover" part), first deconstruct $a \in \widehat{\sigma} P$ as

$$
a=\left(\neg b_{1} \cup c_{1}\right) \cap \cdots \cap\left(\neg b_{n} \cup c_{n}\right)
$$

where $b_{i}$ 's and $c_{i}$ 's are elements of the original $P$. Then

$$
[\mathrm{m}] a=[\mathrm{m}]\left(\neg b_{1} \cup c_{1}\right) \cap \cdots \cap[\mathrm{m}]\left(\neg b_{n} \cup c_{n}\right) .
$$

For each $i,[\mathrm{~m}]\left(\neg b_{i} \cup c_{i}\right)$ is just $b_{i} \underline{-} \underline{3} c_{i} \in P$. The conclusion then follows from the fact $P$ is closed under $\underline{\underline{3}}$ and $\cap$.

A straightforward verification shows that $\widehat{\sigma} f: \widehat{\sigma} \mathfrak{G} \rightarrow \widehat{\sigma} \mathfrak{G}^{\prime}$ is a general S4BHL-frame morphism, whenever $f: \mathfrak{G} \rightarrow \mathfrak{G}^{\prime}$ is a general - -frame morphism, and that $\widehat{\sigma}$ is functorial, so that: 
4.6 Proposition. The assignment $\widehat{\sigma}$ from Definition 4.4 defines a functor $\widehat{\sigma}:$ G-Frm $\rightarrow$ G-S4BHL, and hence also from G-Frm to G-S4K.

Now let us define a functor in the converse direction. For a general S4K-frame $\mathfrak{F}=$ $\left(X, R_{\mathrm{i}}, R_{\mathrm{m}}, P\right)$, let $R_{\mathrm{m}}^{*}=R_{\mathrm{i}} \circ R_{\mathrm{m}}$. (This differs from [109, §2], where Box is enforced by setting $\left.R_{\mathrm{m}}^{*}=R_{\mathrm{i}} \circ R_{\mathrm{m}} \circ R_{\mathrm{i}}.\right)$ Then $\mathfrak{F}^{*}=\left(X, R_{\mathrm{i}}, R_{\mathrm{m}}^{*}, P\right)$ is a general S4BHL-frame. The only thing separating $\left(X, R_{\mathrm{i}}, R_{\mathrm{m}}^{*}\right)$ from a -3 -frame is the fact that $\left(X, R_{\mathrm{i}}\right)$ is allowed to be a pre-order. To resolve this, we quotient out cycles in $X$ :

Let $\mathfrak{F}=\left(X, R_{\mathrm{i}}, R_{\mathrm{m}}, P\right)$ be a general S4BHL-frame. The relation $\sim$ on $X$, given by $x \sim y$ if $x R_{\mathrm{i}} y$ and $y R_{\mathrm{i}} x$ is an equivalence relation on $X$, whose equivalence classes are called $\left(R_{\mathrm{i}}\right.$ )clusters. Let $\lfloor X\rfloor$ be the set of clusters of $\left(X, R_{\mathrm{i}}\right)$, and write $\lfloor x\rfloor$ for the cluster containing $x \in X$. Then $R_{\mathrm{i}}$ defines a partial order on $\lfloor X\rfloor$, which we denote by $\left\lfloor R_{\mathrm{i}}\right\rfloor$. Furthermore, define $\left\lfloor R_{\mathrm{m}}\right\rfloor$ by

$$
\lfloor x\rfloor\left\lfloor R_{\mathrm{m}}\right\rfloor\lfloor y\rfloor \quad \text { iff } \quad x R_{\mathrm{m}} y^{\prime} \text { for some } y^{\prime} \sim y .
$$

Since $x \sim x^{\prime}$ implies $x R_{\mathrm{i}} x^{\prime}$ and $R_{\mathrm{i}} \circ R_{\mathrm{m}}=R_{\mathrm{m}}$ this does not depend on the choice of representative of $\lfloor x\rfloor$.

For a set $a \subseteq X$ let $\lfloor a\rfloor=\{\lfloor x\rfloor \mid x \in a\}$, and define

$$
\lfloor P\rfloor=\{\lfloor a\rfloor \subseteq\lfloor X\rfloor \mid \bigcup\lfloor a\rfloor \in P\} .
$$

Then an easy verification shows that

$$
\lfloor\mathfrak{F}\rfloor=\left(\lfloor X\rfloor,\left\lfloor R_{\mathrm{i}}\right\rfloor,\left\lfloor R_{\mathrm{m}}\right\rfloor,\lfloor P\rfloor\right)
$$

is a general S4BHL-frame. On passing, note that $\lfloor\mathfrak{F}\rfloor$ is a general frame morphic image of $\mathfrak{F}$ whenever $\mathfrak{F}$ satisfies BHL:

4.7 Lemma. If $\mathfrak{F}$ is a general S4BHL-frame then the assignment $x \mapsto\lfloor x\rfloor$ defines a general frame morphism $\mathfrak{F} \rightarrow\lfloor\mathfrak{F}\rfloor$.

Proof. This follows immediately from the construction of $\lfloor\mathfrak{F}\rfloor$.

Finally, we construct a general -3 -frame. Let

$$
\widehat{\rho} P:=\{[i]\lfloor a\rfloor \mid\lfloor a\rfloor \in\lfloor P\rfloor\} .
$$

It follows from $[23, \S 8.3]$ that $\widehat{\rho} P$ is closed under $\cap, \cup$ and $\rightarrow$. Moreover, $\widehat{\rho} P$ is closed under $\underline{-}$ because

$$
[\mathrm{i}] a \underline{-} \underline{\mathrm{i}}] b=[\mathrm{m}](\neg[\mathrm{i}] a \cup[\mathrm{i}] b)=[\mathrm{i}][\mathrm{m}](\neg[\mathrm{i}] a \cup[\mathrm{i}] b) \in \widehat{\rho} P .
$$

Therefore we may define:

4.8 Definition. For a general $\mathrm{S} 4 \mathrm{~K}$ frame $\mathfrak{F}=\left(X, R_{\mathrm{i}}, R_{\mathrm{m}}, P\right)$ define $\widehat{\rho} \mathfrak{F} \in \mathrm{G}-\mathrm{Frm}$ by

$$
\widehat{\rho} \mathfrak{F}=\left(\lfloor X\rfloor,\left\lfloor R_{\mathrm{i}}\right\rfloor,\left\lfloor R_{\mathrm{m}}^{*}\right\rfloor, \widehat{\rho} P\right) .
$$

For a morphism $f: \mathfrak{F} \rightarrow \mathfrak{F}^{\prime}$ in G-S4K define $\widehat{\rho} f: \widehat{\rho} \mathfrak{F} \rightarrow \widehat{\rho} \mathfrak{F}^{\prime}$ by $\widehat{\rho} f(\lfloor x\rfloor)=\lfloor f(x)\rfloor$.

4.9 Proposition. $\hat{\rho}: \mathrm{G}-\mathrm{S} 4 \mathrm{~K} \rightarrow \mathrm{G}-\mathrm{Frm}$ is a functor. 
Proof. We have already seen that $\rho$ is well defined on objects. To see that the same goes for morphisms, let $f:\left(X, R_{\mathrm{i}}, R_{\mathrm{m}}, P\right) \rightarrow\left(X^{\prime}, R_{\mathrm{i}}^{\prime}, R_{\mathrm{m}}^{\prime}, P^{\prime}\right)$ be a morphism in G-S4K and observe that monotonicity of $f$ proves that that $x \sim x^{\prime}$ implies $f(x) \sim f\left(x^{\prime}\right)$, so that the definition of $\rho f$ does not depend on the choice of representative of $\lfloor x\rfloor$. Boundedness of $\rho f$ with respect to both relations is an immediate consequence of the fact that $f$ is a p-morphism. Furthermore, to see that $\rho f$ is a general frame morphism, we need to prove that $(\rho f)^{-1}\left([i]\left\lfloor a^{\prime}\right\rfloor\right) \in \widehat{\rho} P$ for all $a^{\prime} \in P^{\prime}$. This follows from the fact that $(\rho f)^{-1}\left([i]\left\lfloor a^{\prime}\right\rfloor\right)=[i]\left\lfloor f^{-1}\left(a^{\prime}\right)\right\rfloor$.

Functoriality of $\rho$ is straightforward.

4.10 Remark. We can also view $\widehat{\rho}$ and $\widehat{\sigma}$ as acting on non-general frames by viewing a $-3-$ frame as a general -3 -frame where every upset is admissible, and similar for S4K-frames. This observation will be used in the proof of Thm. 4.23 .

Not surprisingly, applying first $\widehat{\sigma}$ and then $\widehat{\rho}$ to a general -3 -frame yields an isomorphic frame. In fact, we can prove that the composition $\widehat{\rho} \widehat{\sigma}$ is naturally isomorphic to the identity functor on G-Frm. We will use this fact to prove facts about modal companions of iA-logics in Thm. 4.16 and Thm. 4.20 (the analogue of the Blok-Esakia theorem).

4.11 Proposition. We have a natural iso $\widehat{\rho} \widehat{\sigma} \cong i d_{\mathrm{G}-\mathrm{Frm}}$.

Proof. Let $\mathfrak{G}=(X, \preceq, \sqsubset, P)$ be a general - -frame. By definition of $\rho$ and $\sigma$ we have $\widehat{\rho} \widehat{\sigma} \mathfrak{G}=$ $(X, \preceq, \sqsubset, \widehat{\rho} \widehat{\sigma} P)$, so for the isomorphism on objects we only have to show that $P=\widehat{\rho} \widehat{\sigma} P$. Since the definition of $\widehat{\rho} \widehat{\sigma} P$ is as in [23, $\S 8.3]$, this follows from Thm. 8.34 in op. cit. Naturality of the isomorphism follows from the fact that $\widehat{\rho} \widehat{\sigma} f=f$.

As in [23, Thm. 8.53], we can prove that $\widehat{\sigma}$ and $\widehat{\rho}$ preserve descriptiveness. This also extends [109, Prop. 7], but requires a more complicated proof.

Descriptive frames are closely connected to Stone spaces, and we will make use of this perspective in the proof of Prop. 4.12. In particular, if $\mathfrak{F}=\left(X, R_{\mathrm{i}}, R_{\mathrm{m}}, P\right)$ is a descriptive S4Kframe and $\tau_{A}$ is topology on $X$ generated by (clopen) base $P$, then $\left(X, \tau_{A}\right)$ is a Stone space, hence compact, and $R_{\mathrm{i}}[x]=\left\{y \in X \mid x R_{\mathrm{i}} y\right\}$ and $R_{\mathrm{m}}[x]=\left\{y \in X \mid x R_{\mathrm{m}} y\right\}$ are closed in $\tau_{A}$ for all $x \in X$. See also [61].

4.12 Proposition. $\widehat{\rho}$ and $\widehat{\sigma}$ preserve descriptiveness.

Proof of Proposition 4.12. Suppose $\mathfrak{G}=(X, \preceq, \sqsubset, P)$ is a descriptive -3 -frame. Then the fact that it is differentiated and compact proves that $\widehat{\sigma} \mathfrak{G}$ is differentiated and compact. Tightness follows from the fact that $\mathfrak{G}$ is a descriptive -3 -frame.

Conversely, suppose $\mathfrak{F}=\left(X, R_{\mathrm{i}}, R_{\mathrm{m}}, P\right)$ is a descriptive S4K-frame. Then $\left(\lfloor X\rfloor,\left\lfloor R_{\mathrm{i}}\right\rfloor, \widehat{\rho} P\right)$ is a descriptive intuitionistic Kripke frame by [23, Thm. 8.53]. So we only have to show that

$$
\begin{aligned}
\lfloor x\rfloor\left\lfloor R_{\mathrm{m}}^{*}\right\rfloor\lfloor y\rfloor \quad \text { iff } & \text { for all }[\mathrm{i}]\lfloor a\rfloor,[\mathrm{i}]\lfloor b\rfloor \in \widehat{\rho} P, \\
& \text { if }\lfloor x\rfloor \in[\mathrm{i}]\lfloor a\rfloor \underline{3}[\mathrm{i}]\lfloor b\rfloor \\
& \text { and }\lfloor y\rfloor \in[i]\lfloor a\rfloor \\
& \text { then }\lfloor y\rfloor \in[i]\lfloor b\rfloor
\end{aligned}
$$

The direction from left to right follows immediately from the definition of -3 , so we focus on the converse.

Suppose $\neg\left(\lfloor x\rfloor\left\lfloor R_{\mathrm{m}}^{*}\right\rfloor\lfloor y\rfloor\right)$. Then $\neg\left(x R_{\mathrm{m}}^{*} y^{\prime}\right)$ for all $y^{\prime} \sim y$. We will construct $a, b \in P$ that are up-closed under $R_{\mathrm{i}}$ such that $y \in a$ and $y \notin b$ and $R_{\mathrm{m}}^{*}[x] \cap a \subseteq b$. (Here $R_{\mathrm{m}}^{*}[x]=\{z \in X \mid$ $\left.x R_{\mathrm{m}}^{*} z\right\}$.) Then, since $a$ and $b$ are up-closed under $R_{\mathrm{i}}$, we have $\bigcup\lfloor a\rfloor=a$ and $\bigcup\lfloor b\rfloor=b$, so that 
$[i]\lfloor a\rfloor,[i]\lfloor b\rfloor \in \hat{\rho} P$. Moreover, by construction [i] $\lfloor a\rfloor$ and [i] $\lfloor b\rfloor$ are such that $\lfloor x\rfloor \in[i]\lfloor a\rfloor \underline{-3}[i]\lfloor b\rfloor$ and $\lfloor y\rfloor \in[i]\lfloor a\rfloor$, while $\lfloor y\rfloor \notin[i]\lfloor b\rfloor$. Therefore, they witness the right-to-left direction in (2).

We view $\mathfrak{F}=\left(X, R_{\mathrm{i}}, R_{\mathrm{m}}, P\right)$ as a Stone space $\left(X, \tau_{P}\right)$ with point-closed relations $R_{\mathrm{i}}$ and $R_{\mathrm{m}}$ (cf. § 4.1). This allows us to use topological in the construction of $a$ and $b$.

So suppose $\neg\left(x R_{\mathrm{m}}^{*} y^{\prime}\right)$ for all $y^{\prime} \sim y$. Then $\uparrow_{\mathrm{m}} x=\left\{z \in X \mid x R_{\mathrm{m}} z\right\}$ and $\downarrow_{\mathrm{i}} y=\left\{z \in X \mid z R_{\mathrm{i}} y\right\}$ are closed, and hence their intersection $C=\uparrow_{\mathrm{m}} x \cap \downarrow_{\mathrm{i}} y$ is closed in $\left(X, \tau_{P}\right)$. Furthermore, by assumption $C$ does not contain any $y^{\prime} \sim y$. Therefore none of the elements in $C$ lies above $y$ in the $R_{\mathrm{i}}$-ordering. We claim that we can find a clopen $R_{\mathrm{i}}$-upset $a$ containing $y$ and disjoint from $\uparrow_{\mathrm{m}} x \cap \downarrow_{\mathrm{i}} y$. To see this, note that since $\uparrow_{\mathrm{i}} y$ is closed we have $\uparrow_{\mathrm{i}} y=\bigcap\left\{a \in P \mid \uparrow_{\mathrm{i}} y \subseteq a\right\}$. Therefore

$$
\begin{aligned}
\uparrow_{\mathrm{i}} y=[\mathrm{i}]\left(\uparrow_{\mathrm{i}} y\right) & =[\mathrm{i}]\left(\bigcap\left\{a \in P \mid \uparrow_{\mathrm{i}} y \subseteq a\right\}\right) \\
& =\bigcap\left\{[\mathrm{i}] a \in P \mid \uparrow_{\mathrm{i}} y \subseteq a\right\} .
\end{aligned}
$$

A compactness argument using compactness of $C$ and the fact that $C$ and $\uparrow_{\mathrm{i}} y$ are disjoint now yields a clopen set $a:=[\mathrm{i}] a_{1} \cap \cdots \cap[\mathrm{i}] a_{n} \in P$ (hence up-closed under $R_{\mathrm{i}}$ ) containing $\uparrow_{\mathrm{i}} y$ and disjoint from $C$.

Similarly, using the fact that $\uparrow_{\mathrm{m}} x \cap a$ is closed and does not contain $y$, we can find a clopen $R_{\mathrm{i}}$-upset $b$ containing $\uparrow_{\mathrm{m}} x \cap a$ such that $y \notin b$. Then we have $y \in[\mathrm{i}] a=a, y \notin[\mathrm{i}] b=b$ and $\uparrow_{\mathrm{m}} x \cap a \subseteq b$, as desired.

\subsection{Modal Companions}

Recall that $t: \mathcal{L}_{3} \rightarrow \mathcal{L}_{\mathrm{i}, \mathrm{m}}$ denotes the extension of the Gödel-McKinsey-Tarski translation of $\mathcal{L}_{-3}$ into classical bimodal logic. The translation $t$ and functor $\hat{\rho}$ are related as follows.

4.13 Lemma. Let $\mathfrak{F} \in \mathrm{G}-\mathrm{S} 4 \mathrm{~K}$ and $\varphi \in \mathcal{L}_{-}$. Then

$$
\mathfrak{F} \Vdash t(\varphi) \quad \text { iff } \quad \widehat{\rho} \mathfrak{F} \Vdash \varphi .
$$

Proof. See Appendix A.3.

This lemma extends Lem. 8.28 in [23], and is the "-3-analogue" of [109, Lem. 5]. It gives rise to the following (standard) notion of modal companions.

4.14 Definition. Let $\Lambda$ be an extension of iA and $\Theta$ an extension of S4K. If for all $\varphi \in \mathcal{L}_{3}$ we have

$$
\varphi \in \Lambda \quad \text { iff } \quad t(\varphi) \in \Theta
$$

then we say that $\Lambda$ is embedded in $\Theta$, and $\Theta$ is an $\mathrm{S} 4 \mathrm{~K}$-companion or modal companion of $\Lambda$.

Analogously to [23, Thm. 9.56], it follows from Lem. 4.13 that for every S4K-logic $\Theta$, the iA-logic

$$
\rho \Theta=\left\{\varphi \in \mathcal{L}_{-3} \mid t(\varphi) \in \Theta\right\}
$$

is such that $\Theta$ is an S4K-companion of $\rho \Theta$. Furthermore, it follows from Lem. 4.13 that:

4.15 Proposition. If an $\mathrm{S} 4 \mathrm{~K}-$ logic $\Theta$ is characterised by a class $\mathcal{C}$ of general S4K-frames, then $\rho \Theta$ is characterised by the class $\widehat{\rho} \mathfrak{C}=\{\widehat{\rho} \mathfrak{F} \mid \mathfrak{F} \in \mathcal{C}\}$ of general -3-frames.

The proof of the following theorem resembles to proof of [109, Thm. 9], using Lem. 4.13 and Prop. 4.11. 
4.16 Theorem. Each $\mathrm{iA}$-logic $\Lambda=\mathrm{iA} \oplus \Gamma$ is embeddable by $t$ in any logic $\Theta$ in the interval

$$
\left[(\mathrm{S} 4 \otimes \mathrm{K}) \oplus t(\Gamma),\left(\mathrm{Grz}_{\mathbf{i}} \otimes \mathrm{K}\right) \oplus t(\Gamma) \oplus \mathrm{BHL}\right] .
$$

We have seen how S4K-logics give rise to iA-logics. In the converse direction, guided by Thm. 4.16, we define:

4.17 Definition. For an iA-logic $\Lambda=\mathrm{iA} \oplus \Gamma$, let

$$
\begin{aligned}
\tau \Lambda & =(\mathrm{S} 4 \otimes \mathrm{K}) \oplus t(\Gamma) \oplus \mathrm{BHL} \\
\sigma \Lambda & =\left(\mathrm{Grz}_{\mathrm{i}} \otimes \mathrm{K}\right) \oplus t(\Gamma) \oplus \mathrm{BHL}
\end{aligned}
$$

\subsection{Blok-Esakia Theorem}

Finally, we work our way towards an analogue of the Blok-Esakia theorem. Recall that the Blok-Esakia theorem states that an S4-logic $\Theta$ is a modal companion of an intuitionistic logic $\Lambda$ if and only if $\tau \Lambda \subseteq \Theta \subseteq \sigma \Lambda$. We prove that, with our extended definitions of $\tau$ and $\sigma$, this statement still holds.

Before we can prove the -3 -analogue of the Blok-Esakia theorem, we need two more lemmas. These are counterparts of Lem. 25 and 26 in [108].

4.18 Lemma. Let $\mathfrak{G}=\left(X, R_{\mathrm{i}}, R_{\mathrm{m}}, P\right)$ be an $\mathrm{S} 4 \mathrm{BHL}$-frame such that $R_{\mathrm{i}}$ is a partial order and $\mathfrak{G} \Vdash \mathrm{Grz}_{\mathrm{i}}$. Then for all $\varphi \in \mathcal{L}_{\mathrm{i}, \mathrm{m}}$ we have $\mathfrak{G} \Vdash \varphi$ iff $\widehat{\sigma} \widehat{\rho} \mathfrak{G} \Vdash \varphi$.

Proof. This can be proved via an adaptation of [108, Lem. 25], which can be found in Appendix A.4.

4.19 Lemma. Every $\mathrm{S} 4 \mathrm{~K}$-logic is characterised by a general $\mathrm{S} 4 \mathrm{~K}$-frame $\left(X, R_{\mathrm{i}}, R_{\mathrm{m}}, P\right)$ such that $R_{\mathrm{i}}$ is a partial order.

Proof. See Appendix A.5.

The following is an analogue of the Blok-Esakia theorem.

4.20 Theorem. An $\mathrm{S} 4 \mathrm{~K}$-logic $\Theta$ containing $\mathrm{BHL}$ is an $\mathrm{S} 4 \mathrm{~K}$-companion of an $\mathrm{A} \mathrm{A}-$ logic $\Lambda$ if and only if $\tau \Lambda \subseteq \Theta \subseteq \sigma \Lambda$.

Proof of Theorem 4.20. The direction from right to left follows from Thm. 4.16. For the converse it suffices to show that $\Theta \subseteq \sigma \Lambda$.

Following the structure of the proof of [108, Thm. 27], we first establish

$$
\left\{(\widehat{\rho} \mathfrak{F})^{*} \mid \mathfrak{F} \Vdash \Theta\right\}=\left\{\mathfrak{G}^{*} \mid \mathfrak{G} \Vdash \Lambda\right\} .
$$

(Recall that $\mathfrak{F}^{*}$ denotes dual HL-algebra of $\mathfrak{F}$, see $\S 3.3$.)

By Prop. 4.15, $\Lambda$ is characterised by the class $\mathcal{C}=\left\{(\widehat{\rho} \mathfrak{F})^{*} \mid \mathfrak{F} \Vdash \Theta\right\}$, so the left hand side of (3) is contained in the right hand side. In order to prove equality, by Birkhoff's variety theorem it suffices to prove that $\mathcal{C}$ is a variety, i.e., it is closed under direct products, subalgebras and homomorphic images. The first follows from the fact that $\prod_{i \in I}\left(\widehat{\rho} \widetilde{\mathfrak{F}}_{i}\right)^{*}=\left(\widehat{\rho}\left(\coprod_{i \in I} \mathfrak{F}_{i}\right)\right)^{*}$ and $\mathfrak{F}_{i} \Vdash \Theta$ for all $i \in I$ implies $\coprod \mathfrak{F}_{i} \Vdash \Theta$.

Next, suppose that $\mathcal{A}$ is a subalgebra of $(\widehat{\rho} \mathfrak{F})^{*} \in \mathcal{C}$, where $\mathfrak{F}=\left(X, R_{\mathrm{i}}, R_{\mathrm{m}}, P\right)$. Since $\mathfrak{F}$ is assumed to validate BHL, it follows from Lem. 4.7 that we have a surjective general frame morphism $\mathfrak{F} \rightarrow\lfloor\mathfrak{F}\rfloor$, so that $\lfloor F\rfloor \Vdash \Theta$. Write $A$ for the carrier of $\mathcal{A}$. Then the general 3 frame $\mathfrak{A}:=\left(\lfloor X\rfloor,\left\lfloor R_{\mathrm{i}}\right\rfloor,\left\lfloor R_{\mathrm{m}}\right\rfloor, A\right)$ is a homomorphic image of $\widehat{\rho} \mathfrak{F}$ and by construction $\widehat{\sigma} \mathfrak{A}=$ 
$\left(\lfloor X\rfloor,\left\lfloor R_{\mathrm{i}}\right\rfloor,\left\lfloor R_{\mathrm{m}}\right\rfloor, \sigma A\right)$ is a homomorphic image of $\lfloor\mathfrak{F}\rfloor$, so that $\widehat{\sigma} \mathfrak{A} \Vdash \Theta$. By construction of $\mathcal{A}$ and Prop. 4.11 we then have $\mathcal{A}=(\widehat{\rho} \widehat{\sigma} \mathfrak{A})^{*}$. Therefore $\mathcal{A} \in \mathcal{C}$.

To prove that $\mathcal{C}$ is closed under homomorphic images, suppose that $\mathfrak{F}=\left(X, R_{\mathrm{i}}, R_{\mathrm{m}}, P\right)$ is an S4BHL-frame for $\Theta$ and $h$ a homomorphism from $(\widehat{\rho} \mathfrak{F})^{*}$ onto $\mathfrak{H}^{*}$, where $\mathfrak{H}$ is some general -3 frame. Since $(\widehat{\sigma} \widehat{\rho} \mathfrak{F})^{*}$ is a subalgebra of $\mathfrak{F}^{*}$ we have $(\widehat{\sigma} \widehat{\rho} \mathfrak{F})^{*} \Vdash \Theta$. As a consequence of Prop. 4.11 we have $(\widehat{\rho} \widehat{\sigma} \mathfrak{H})^{*}=\mathfrak{H}^{*}$, so it is sufficient to construct a homomorphism $g$ from $(\widehat{\sigma} \widehat{\rho} \mathfrak{F})^{*}$ onto $(\widehat{\sigma} \mathfrak{H})^{*}$, because this implies $\widehat{\sigma} \mathfrak{H} \Vdash \Theta$.

Every set $a \in \widehat{\sigma} \widehat{\rho} P$ can be represented as

$$
a=\left(-b_{1} \cup c_{1}\right) \cap \cdots \cap\left(-b_{n} \cup c_{n}\right),
$$

where $b_{i}, c_{i} \in \widehat{\rho} P$. Define $g:(\widehat{\sigma} \widehat{\rho} \mathfrak{F})^{*} \rightarrow(\widehat{\sigma} \mathfrak{H})^{*}$ via

$$
g(a)=\left(-h\left(b_{1}\right) \cup h\left(c_{1}\right)\right) \cap \cdots \cap\left(-h\left(b_{n}\right) \cup h\left(c_{n}\right)\right) .
$$

Then $g(a) \in(\widehat{\sigma} \mathfrak{H})^{*}$, and if $a \in \widehat{\rho} P$ then $g(a)=h(a)$. It was shown in [75, Lem. 3.3] that $g$ is a surjection and preserves the Boolean operations and $\square_{\mathrm{i}}$.

Let us show that it preserves $\square_{\mathrm{m}}$ as well. Write $a$ as above. Since $g$ and $\square_{\mathrm{m}}$ preserve finite meets we have

$$
g\left(\square_{\mathrm{m}} a\right)=g\left(\square_{\mathrm{m}} \bigcap_{i=1}^{n}\left(-b_{i} \cup c_{i}\right)\right)=\bigcap_{i=1}^{n} g\left(\square_{\mathrm{m}}\left(-b_{i} \cup c_{i}\right)\right) .
$$

Furthermore, $\square_{\mathrm{m}}\left(-b_{i} \cup c_{i}\right)=b_{i}-3 c_{i} \in \widehat{\rho} P$ for all $b_{i}, c_{i} \in \widehat{\rho} P$, and $h$ preserves elements in $\widehat{\rho} P$, so that

$$
\begin{aligned}
g\left(\square_{\mathrm{m}}\left(-b_{i} \cup c_{i}\right)\right) & =g\left(b_{i} \underline{3} c_{i}\right)=h\left(b_{i} \underline{-3} c_{i}\right) \\
& =h\left(b_{i}\right) \underline{-3} h\left(c_{i}\right)=g\left(b_{i}\right) \underline{-3} g\left(c_{i}\right) \\
& =\square_{\mathrm{m}}\left(-g\left(b_{i}\right) \cup g\left(c_{i}\right)\right) .
\end{aligned}
$$

Consequently, we obtain

$$
\begin{aligned}
g\left(\square_{\mathrm{m}} a\right) & =\bigcap_{i=1}^{n} g\left(\square_{\mathrm{m}}\left(-b_{i} \cup c_{i}\right)\right) \\
& =\bigcap_{i=1}^{n} \square_{\mathrm{m}}\left(-g\left(b_{i}\right) \cup g\left(c_{i}\right)\right) \\
& =\square_{\mathrm{m}} \bigcap_{i=1}^{n}\left(-g\left(b_{i}\right) \cup g\left(c_{i}\right)\right) \\
& =\square_{\mathrm{m}} g(a)
\end{aligned}
$$

So (3) holds.

To prove that $\Theta \subseteq \sigma \Lambda$, it suffices to show that a characteristic frame $\mathfrak{F}=\left(X, R_{\mathrm{i}}, R_{\mathrm{m}}, P\right)$ for $\sigma \Lambda$ is also a frame for $\Theta$. By Lem. 4.19 we may assume that $R_{\mathrm{i}}$ is a partial order. Since $\widehat{\rho} \mathfrak{F} \Vdash \Lambda$, it follows from Equation (3) that there exists a $\mathfrak{F}^{\prime}$ for $\Theta$ such that $\widehat{\rho} \mathfrak{F} \simeq \widehat{\rho} \mathfrak{F}^{\prime}$ and so $\widehat{\sigma} \widehat{\rho} \mathfrak{F} \simeq \widehat{\sigma} \widehat{\rho} \mathfrak{F}^{\prime}$. Since $\widehat{\sigma} \widehat{\rho} \mathfrak{F}^{\prime} \Vdash \Theta$ this implies $\widehat{\sigma} \widehat{\rho} \mathfrak{F} \Vdash \Theta$ and it follows from Lem. 4.18 that $\mathfrak{F} \Vdash \Theta$.

As a corollary of Thm. 4.20, we obtain:

\subsection{Corollary.}


1) Let $\Lambda$ be an iA-logic. Then $\tau \Lambda$ and $\sigma \Lambda$ are the smallest and greatest modal companions of $\mathcal{L}$, respectively.

2) The map $\sigma$ is an isomorphism from the lattice of $\mathrm{iA}$-logics to the lattice of $\left(\mathrm{S} 4 \mathrm{BHL} \oplus \mathrm{Grz}_{\mathrm{i}}\right)$ logics.

We can prove the following analogue of Prop. 4.15, which characterises a logic of the form $\sigma \Lambda$.

4.22 Proposition. If an $\mathrm{iA-logic} \Lambda$ is characterised by a class $\mathcal{C}$ of general -3 -frames, then the logic $\sigma \Lambda$ is characterised by $\widehat{\sigma} \mathfrak{C}=\{\widehat{\sigma} \mathfrak{G} \mid \mathfrak{G} \in \mathcal{C}\}$.

Proof. The proof is analogous to [108, Prop. 29], using Lem. 4.13 and Prop. 4.11 and Thm. 4.20.

Finally, let us summarise how the developed theory allows us to transfer results between iA-logics and S4K-logics.

\subsection{Theorem.}

1) The map $\rho$ preserves decidability, Kripke completeness and the finite model property.

2) The map $\rho$ preserves canonicity of S4BHL-logics.

3) The map $\tau$ preserves canonicity.

4) The map $\sigma$ preserves the finite model property.

Proof. The first and fourth item follows from the definition of $\rho$, Propositions 4.15 and 4.22, and the fact that $\widehat{\rho} \mathfrak{G}$ and $\widehat{\sigma} \mathfrak{F}$ are finite whenever $\mathfrak{G}$ and $\mathfrak{F}$ are.

For the second, suppose $\Theta$ is an S4BHL-logic, i.e., an S4K-logic containing BHL. If $\mathfrak{G}$ is a descriptive frame for $\rho \Theta$, then by Prop. 4.12 and Thm. $4.20 \widehat{\rho} \mathfrak{G}$ is a descriptive frame for $\sigma \rho M$. Since $\Theta$ is canonical we have $\kappa \widehat{\sigma} \mathfrak{G} \Vdash \Theta$, where $\kappa \widehat{\sigma} \mathfrak{G}$ denotes the (non-general) S4K-frame underlying $\widehat{\sigma} \mathfrak{G}$. Therefore $\widehat{\rho} \kappa \widehat{\sigma} \mathfrak{G} \Vdash \rho \Theta$. Inspection of the definitions of $\widehat{\rho}$ and $\widehat{\sigma}$ show that $\widehat{\rho} \kappa \widehat{\sigma} \mathfrak{G}=\kappa \mathfrak{G}$, which completes the proof.

Third, if $\mathfrak{F}$ is a descriptive frame for $\tau \Lambda$, then by Propositions 4.12 and 4.15 and Thm. 4.20 $\widehat{\rho} \mathfrak{F}$ is a descriptive frame for $\rho \tau \Lambda=\Lambda$. It then follows from Lem. 4.13 and the observation that $\kappa \widehat{\rho} \mathfrak{F}=\widehat{\rho} \kappa \mathfrak{F}$ that $\kappa \mathfrak{F} \Vdash \tau \Lambda$.

4.24 Example. 1. A modal companion of Box is given by the extension of $\mathrm{S} 4 \mathrm{~K}$ with $\square_{\mathrm{i}} \square_{\mathrm{m}} \square_{\mathrm{i}} p \rightarrow$ $\square_{\mathrm{m}} p$. This axiom is canonical and elementary via the SQEMA algorithm [27,44] (or the usual Sahlqvist argument), which also yields strong completeness.

2. The translation of $\mathrm{P}$ from $\S 2.5$ is $\mathrm{S} 4 \mathrm{BHL}$-equivalent to $\square_{\mathrm{m}}\left(\square_{\mathrm{i}} p \rightarrow \square_{\mathrm{i}} q\right) \rightarrow \square_{\mathrm{m}} \square_{\mathrm{m}}\left(\square_{\mathrm{i}} p \rightarrow\right.$ $\square_{i} q$ ). Here we need to be somewhat creative. Namely, we observe that by the results of this section, if $\tau \Lambda$ is canonical, then $\Lambda$ is strongly complete with respect to $\tau \Lambda$-frames, which are in addition partial orders. Next, one observes that over partial orders, the following rule is admissible: from $\varphi\left(\square_{\mathbf{i}} p \rightarrow \square_{\mathbf{i}} q\right)$, derive $\varphi(r)$, where $p$ and $q$ are fresh for $\varphi(r)$ The above translation can be verified to be canonical via the SQEMA algorithm $[27,44]$. In this way, we finally arrive at $R_{\mathrm{m}}$-transitivity as its (canonical) counterpart (see also $[74, \S 10])$. 
3. The translation of IR reads

$$
\square_{\mathrm{i}}\left(\square_{\mathrm{i}} \square_{\mathrm{m}}\left(\square_{\mathrm{i}} p \rightarrow \square_{\mathrm{i}} q\right) \rightarrow \square_{\mathrm{i}}\left(\square_{\mathrm{i}} p \rightarrow \square_{\mathrm{i}} \diamond_{\mathrm{i}} \square_{\mathrm{i}} q\right)\right),
$$

where $\diamond_{i}$ is short for $\neg \square_{i} \neg$. As a consequence of Thm. 4.16 the logic $\tau$ IELE $=$ S4K $\oplus$ $t\left(\mathrm{~S}_{\square}\right) \oplus t(\mathrm{IR})$ is a modal companion of IELE.

\section{A Criterion for FMP and Decidability}

Clause 1) of Thm. 4.23 allows transfer of decidability and the finite model property from a bimodal logic to its -3-counterpart. Recall that for a finitely axiomatizable logic, the finite model property is a sufficient criterion of decidability. Still, in order to use this theorem for a -3logic, we need a bimodal counterpart which enjoys these properties. Wolter and Zakharyaschev [109] proposed a broad criterion based on techniques for unimodal (cofinal) subframe logics. We begin by generalizing their criterion to S4BHL-logics. They essentially relies on transitivity of both relations: not only $R_{\mathrm{i}}$, but also $R_{\mathrm{m}}$. Logically, the latter corresponds to validity of

$$
4_{\mathrm{m}} \square_{\mathrm{m}} p \rightarrow \square_{\mathrm{m}} \square_{\mathrm{m}} p
$$

That is, we will be concerned with ( $\mathrm{S} 4 \otimes \mathrm{K} 4)$-logics. In the presence of the Mix-axiom, i.e.,

$\operatorname{Mix} \square_{\mathrm{m}} p \rightarrow \square_{\mathrm{i}} \square_{\mathrm{m}} \square_{\mathrm{i}} p$

the original criterion reads as

5.1 Theorem ([109], Thm. 17). Let $\Theta$ be a canonical subframe extension of S4K containing Mix. If $\mathrm{S} 4 \oplus \Gamma \subseteq \mathcal{L}_{\mathrm{i}}$ is a $R_{\mathrm{i}}$-subframe logic, then $\Theta \oplus \Gamma$ has the finite model property.

The goal of this section is to prove similar theorems that do not require Mix. First, recall that the subframe of $\mathfrak{F}=\left(X, R_{\mathrm{i}}, R_{\mathrm{m}}, P\right)$ generated by $Y \subseteq X$ is the frame $\mathfrak{F}_{\uparrow Y}=$ $\left(Y, R_{\mathrm{i} \uparrow Y}, R_{\mathrm{m} \uparrow Y}, P_{\uparrow Y}\right)$, where $R_{\mathrm{i} \uparrow Y}$ and $R_{\mathrm{m} \uparrow Y}$ are the restrictions of $R_{\mathrm{i}}$ and $R_{\mathrm{m}}$ to $Y$, and $P_{\uparrow Y}=\{a \cap Y \mid a \in P\}$. It is called $R_{\mathrm{m}}$-cofinal if for all $y \in Y, y R_{\mathrm{m}} z$ implies that there exists $y^{\prime} \in Y$ such that $z R_{\mathrm{m}} y^{\prime}$. An S4K-logic $\Theta$ is called a ( $R_{\mathrm{m}}$-cofinal) subframe logic if the collection of frames validating $\Theta$ is closed under forming $\left(R_{\mathrm{m}}\right.$-cofinal) subframes.

Besides, we make heavy use of the notions of $R_{\mathrm{i}}$ - and $R_{\mathrm{m}}$-maximality with respect to an equivalence relation generated by a formula $\varphi$ :

5.2 Definition. Let $\mathfrak{M}=(\mathfrak{F}, V)$ be a model based on a general S4K-frame $\mathfrak{F}=\left(X, R_{\mathrm{i}}, R_{\mathrm{m}}, P\right)$. If $x \in X$ then we write $\uparrow_{\mathrm{i}} x=\left\{y \in X \mid x R_{\mathrm{i}} y\right\}$ for the upwards closure of $x$ and $C_{\mathrm{i}}(x)=\{y \in X \mid$ $x \leq y \leq x\}$ for the $R_{\mathrm{i}}$-cluster of $x$. The state $x$ is called $R_{\mathrm{i}}$-final, and $C(x)$ is called an $R_{\mathrm{i}}$-final $R_{\mathrm{i}}$-cluster, if $\uparrow_{\mathrm{i}} x=C_{\mathrm{i}}(x)$. We similarly define $\left(R_{\mathrm{m}}\right.$-final $) R_{\mathrm{m}}$-clusters.

Let $\varphi \in \mathcal{L}_{\mathrm{i}, \mathrm{m}}$. We call $x, y \in X \varphi$-equivalent in $\mathfrak{M}$, and write $x \sim_{\varphi} y$, if $x \Vdash \psi$ iff $y \Vdash \psi$ for all $\psi \in \operatorname{Subf}(\varphi)$. A state $x$ is called $R_{\text {- }}$-maximal in $\mathfrak{M}$ (relative to $\sim_{\varphi}$ ) if for any $x \neq y \in X$ such that $x R_{\mathrm{i}} y$ we have $x \not_{\varphi} y$. We similarly define $R_{\mathrm{m}}$-maximality.

If $\mathfrak{M}$ is based on a descriptive frame, then for every state $x \in \mathfrak{M}$ there exists an $R_{\mathrm{i}}$-maximal state $y$ such that $x \sim_{\varphi} y$ and either $x=y$ or $x R_{\mathrm{i}} y$ [109, Lem. 14]. If $R_{\mathrm{m}}$ is transitive then the same holds for $R_{\mathrm{m}}$.

The purpose of $\varphi$-equivalence is showcased in the following lemma, that will prove useful in the subsequent theorem. 
5.3 Lemma. Let $\mathfrak{M}=(\mathfrak{F}, V)$ be a model based on an $\mathrm{S} 4 \mathrm{~K}$-frame $\mathfrak{F}=\left(X, R_{\mathrm{i}}, R_{\mathrm{m}}, P\right)$, and let $\varphi \in \mathcal{L}_{\mathrm{i}, \mathrm{m}}$. Suppose $Y \subseteq X$ is such that for $R \in\left\{R_{\mathrm{i}}, R_{\mathrm{m}}\right\}$ : if $y \in Y$ and $y R x$ (where $x \in X$ ), then there exists $y^{\prime} \in Y$ such that $y R y^{\prime}$ and $x \sim_{\varphi} y^{\prime}$. Then for all $y \in Y$ and $\psi \in \operatorname{Subf}(\varphi)$ we have

$$
\mathfrak{M}_{\uparrow Y}, y \Vdash \psi \quad \text { iff } \quad \mathfrak{M}, y \Vdash \psi .
$$

Proof. By induction on the structure of $\psi$. If $\psi=p, \top$ or $\perp$ then the statement is obvious, as is the case for $\varphi=\psi_{1} \wedge \psi_{2}$ and $\psi=\psi_{1} \vee \psi_{2}$.

If $\psi=\square_{\mathbf{i}} \psi_{1}$ and $\mathfrak{M}, y \Vdash \square_{\mathbf{i}} \psi_{1}$, then clearly $\mathfrak{M}_{\uparrow Y}, y \Vdash \square_{\mathbf{i}} \psi_{1}$, since every $\left(R_{\mathbf{i} \uparrow Y}\right)$-successor of $y$ is also an $R_{\mathrm{i}}$-successor of $y$ in $\mathfrak{M}$. Conversely, if $\mathfrak{M}, y \Downarrow \square_{\mathrm{i}} \psi_{1}$, then there is an $R_{\mathfrak{i}}$-successor $z$ such that $\mathfrak{M}, z \forall \psi_{1}$. By assumption there exists $z^{\prime} \in Y$ such that $y R_{\mathrm{i}} z^{\prime}$ and $z \sim_{\varphi} z^{\prime}$. Consequently $\mathfrak{M}, z^{\prime} \| \psi_{1}$, so by the induction hypothesis $\mathfrak{M}_{\uparrow Y}, z^{\prime} \| \psi_{1}$ and hence $\mathfrak{M}_{\uparrow Y}, y \| \square_{\mathrm{i}} \psi_{1}$.

The case $\psi=\square_{\mathrm{m}} \psi_{1}$ is analogous.

5.4 Theorem. Suppose $\Theta$ is a canonical extension of $\mathrm{S} 4 \otimes \mathrm{K} 4$ containing $\mathrm{BHL}$ that is closed under forming $\left(R_{\mathrm{m}}\right.$-cofinal) subframes. Then:

1) $\Theta$ has the finite model property.

2) If moreover $\Theta$ contains the classical strength axiom

$$
\mathrm{S}_{\mathrm{c}} \square_{\mathrm{i}} p \rightarrow \square_{\mathrm{m}} p .
$$

then for any $\left(R_{\mathrm{m}}\right.$-cofinal) subframe logic $\Gamma \subseteq \mathcal{L}_{\mathrm{m}}$, the logic $\Theta \oplus \Gamma$ has the finite model property.

Proof. See Appendix A.6.

5.5 Corollary. Let $\Lambda$ be a-3-logic extending i-P.

1) If its S4BHL-counterparts include a canonical logic preserved by forming (cofinal) subframes, $\Lambda$ has the finite model property.

2) Furthermore, if $\Lambda$ extends $\mathrm{i}_{-} \mathrm{S}_{\mathrm{a}}$ and its $\mathrm{S} 4 \mathrm{BHL}$-counterparts include a logic obtained by extending a canonical (cofinal) subframe logic with a collection of $\mathcal{L}_{\mathrm{m}}$-axioms preserved by $R_{\mathrm{m}}$-subframes, $\Lambda$ has the finite model property.

In either case, $\Lambda$ is decidable whenever finitely axiomatizable.

\subsection{Examples.}

- The above theorem covers i-P and i-S $\mathrm{S}_{\mathrm{a}}$ themselves. As we have seen, their natural S4BHL couterparts are complete with respect to frames definable by universal first-order conditions, hence they are not only canonical, but also preserved under subframes.

- It appears more challenging to use the second clause of the above corollary, as the GMT translation always includes some $\square_{i}$ modalities. However, transformations similar to those used in Exm. 4.24 can handle, e.g., a syntactic variant of the strong Löb axiom

$$
(((p-\neg q) \wedge p) \dashv q) \rightarrow(p-\neg q) .
$$

After the GMT translation, one application of the trick from Exm. 4.24 .2 (plus some trivial book-keeping) yields Löb for $\square_{\mathrm{m}}$. This is a $R_{\mathrm{m}}$-subframe axiom. 
- Simlarly, when one considers PLAA, its Appa axiom over i-Sa translates to

$$
\square_{\mathrm{m}}\left(\left(\square_{\mathrm{i}} p \wedge \square_{\mathrm{m}}\left(\square_{\mathrm{i}} p \rightarrow \square_{\mathrm{i}} q\right)\right) \rightarrow \square_{\mathrm{i}} q\right) .
$$

The usual currying trick yields

$$
\left.\square_{\mathrm{m}}\left(\square_{\mathrm{m}}\left(\square_{\mathrm{i}} p \rightarrow \square_{\mathrm{i}} q\right)\right) \rightarrow\left(\square_{\mathrm{i}} p \rightarrow \square_{\mathrm{i}} q\right)\right),
$$

which in one application of the trick from Exm. 4.24.2 produces $\square_{\mathrm{m}}\left(\square_{\mathrm{m}} r \rightarrow r\right)$ and this is a $R_{\mathrm{m}}$-subframe axiom.

\section{Conclusions and Future Work}

We have investigated the Heyting-Lewis (family of) logic(s) of strict implication. We have described suitable categorical duality, have provided a (truth preserving) translation into classical bimodal logic, and have established a Blok-Esakia style theorem for Heyting-Lewis logic. The latter yields results on canonicity, correspondence, the fmp, and decidability.

Our study leaves many questions open, including the limits of the finite model property results discussed in Section $\S 5$. Another challenge is a detailed investigation of the Intuitionistic Logic of Entailment IELE proposed in $\S 2.4$. Apart from its mathematical and philosophical aspects, or the question of full axiomatization capturing all justifiable principles, we note the Brouwer-Heyting-Kolmogorov motivation underlying the original IEL [5]. Here, one could relate it to type-theoretic calculi developed in the functional programming setting (cf. § 2.3). Finally, it would be of interest to compare IELE with other approaches to intuitionistic epistemic logics $[62,90,107]$.

On the more conceptual side, we are interested in viewing Heyting-Lewis logic from a dialgebra perspective [47], in particular as the semantics used in this paper naturally lends itself to a dialgebraic treatment. Dialgebraic methods can, for example, provide a generic expressivity and expressivity-somewhere-else results [47, § 7] and Goldblatt-Thomason theorems [30]. It would be intriguing to see whether dialgebraic methods can provide an elegant connection (say, via a dialgebraic generalisation of the final coalgebra sequence) with, on the one hand, finitary completeness proofs developed in the preservativity community $[51,53]$ and on the other hand, step-algebras and step-frames [13].

But what we see as the main future challenge is the development of semantics and decidability results of $\mathrm{iA}^{-}$logics not including $\mathrm{Di}$. We have seen that such logics arise under the functional programming interpretation (arrows without choice), and also under the arithmetical interpretation (e.g., preservativity without provable closure under q-realizability). ${ }^{10}$ Let us note here that $\mathrm{iA}^{-}$extends the minimal system ICK of intuitionistic conditional logic proposed recently by Weiss $[25,105,106]$, which allows the use of an intuitionistic variant of Chellas frames in conditional logic [24]. However, we believe that the right approach is to generalize so-called Veltman semantics of classical interpretability logics [32], as classically preservativity is the contrapose of arithmetical interpretability. Conceivable variants of generalized Veltman semantics would be equivalent to subclasses of Chellas-Weiss frames (much like Kripke frames can be seen as a limiting case of neighbourhood frames). Nevertheless, in the classical setting,

\footnotetext{
${ }^{10}$ Especially in the functional programming world, it is common to consider fragments not involving disjunction, so the reader may ask if for such formulae at least, one cannot use Kripke completeness for an extension of a disjunction-free set of axioms with $\mathrm{Di}$; in other words, if extensions with $\mathrm{Di}$ are conservative over the disjunctionfree fragment. While the potential scope of such results is of independent interest, the general answer is on the negative [71, Lem. 4.6], [74, § 10].
} 
(generalized) Veltman semantics has proved particularly suitable for decidability and complexity results [32,79,80], allowing adaptations of standard modal techniques such as filtration [89], so it does seem promising to work with more restrictive structures. 


\section{References}

[1] Andreas Abel and Andrea Vezzosi. A formalized proof of strong normalization for guarded recursive types. In Jacques Garrigue, editor, Proc. of APLAS, volume 8858 of LNCS, pages 140-158. Springer International Publishing, 2014.

[2] Natasha Alechina, Michael Mendler, Valeria de Paiva, and Eike Ritter. Categorical and kripke semantics for constructive S4 modal logic. In Laurent Fribourg, editor, Proc. CSL 2001, volume 2142 of Lecture Notes in Computer Science, pages 292-307. Springer, 2001.

[3] Mohammad Ardeshir and Mojtaba Mojtahedi. The $\sigma_{1}$-provability logic of HA*. J. Symb. Log., 84(3):1118-1135, 2019.

[4] Mohammad Ardeshir and S. Mojtaba Mojtahedi. The $\sigma_{1}$ provability logic of HA. Ann. Pure Appl. Log., 169(10):997-1043, 2018.

[5] S. Artemov and T. Protopopescu. Intuitionistic epistemic logic. The Review of Symbolic Logic, 9(2):266-298, 2016.

[6] Robert Atkey. What is a categorical model of arrows? ENTCS, 229(5):19 - 37, 2011. Proceedings of the Second Workshop on Mathematically Structured Functional Programming (MSFP 2008).

[7] Robert Atkey and Conor McBride. Productive coprogramming with guarded recursion. In Greg Morrisett and Tarmo Uustalu, editors, International Conference on Functional Programming, (ICFP), pages 197-208. ACM SIGPLAN, 2013.

[8] Oskar Becker. Zur Logik der Modalitäten. Jahrbuch für Philosophie und phänomenologische Forschung. Halle, 1930.

[9] N. Benton, G.Bierman, and V. De Paiva. Computational types from a logical perspective. Journal of Functional Programming, 8(2):177-193, 1998.

[10] Nick Benton and Nicolas Tabareau. Compiling functional types to relational specifications for low level imperative code. In Andrew Kennedy and Amal Ahmed, editors, Types in Languages Design and Implementation (TLDI), pages 3-14. ACM SIGPLAN, 2009.

[11] Claudio Bernardi. The fixed-point theorem for diagonalizable algebras. Studia Logica, 34(3):239$251,1975$.

[12] N. Bezhanishvili. Lattices of intermediate and cylindric modal logics. PhD thesis, University of Amsterdam, 2006.

[13] N. Bezhanishvili and S. Ghilardi. The bounded proof property via step algebras and step frames. Annals of Pure and Applied Logic, 165(12):1832-1863, 2014.

[14] G. M. Bierman and V. C. V. de Paiva. On an intuitionistic modal logic. Studia Logica: An International Journal for Symbolic Logic, 65(3):383-416, 2000.

[15] Lars Birkedal, Rasmus Ejlers Møgelberg, Jan Schwinghammer, and Kristian Støvring. First steps in synthetic guarded domain theory: Step-indexing in the topos of trees. LMCS, 8:1-45, 2012.

[16] P. Blackburn, M. de Rijke, and Y. Venema. Modal Logic. Cambridge Tracts in Theoretical Computer Science. Cambridge University Press, Cambridge, 2001.

[17] W. J. Blok. Varieties of interior algebras. PhD thesis, University of Amsterdam, 1976.

[18] W. J. Blok and D. Pigozzi. Algebraizable logics, volume 77 (396) of Memoirs AMS. AMS, 1989.

[19] G. Boolos and G. Sambin. Provability: the emergence of a mathematical modality. Stud. Logica, 50:1-23, 1991.

[20] George Boolos. The Logic of Provability. Cambridge University Press, 1993.

[21] M. Božić and K. Došen. Models for normal intuitionistic modal logics. Studia Logica, 43:217-245, 1984.

[22] S. Celani and R. Jansana. Bounded distributive lattices with strict implication. Mathematical Logic Quarterly, 51:219-246, 2005.

[23] A. Chagrov and M. Zakharyaschev. Modal Logic. Oxford University Press, Oxford, 1997.

[24] B. F. Chellas. Modal Logic: An Introduction. Cambridge University Press, Cambridge, 1980. 
[25] I. Ciardelli and X. Liu. Intuitionistic conditional logics. Journal of Philosophical Logic, 2019.

[26] Ranald Clouston and Rajeev Goré. Sequent calculus in the topos of trees. In Andrew M. Pitts, editor, Proc. of FoSSaCS, volume 9034 of LNCS, pages 133-147. Springer, 2015.

[27] Willem Conradie, Valentin Goranko, and Dimiter Vakarelov. Algorithmic correspondence and completeness in modal logic. i. the core algorithm sqema. Logical Methods in Computer Science, 2(1), 2006.

[28] Haskell B. Curry. The elimination theorem when modality is present. J. Symb. Log., 17(4):249265, 1952.

[29] Rowan Davies and Frank Pfenning. A modal analysis of staged computation. J. ACM, 48(3):555$604,2001$.

[30] J. de Groot. Goldblatt-thomason theorems for modal intuitionistic logics, 2020.

[31] D.H.J. de Jongh and A. Visser. Embeddings of Heyting algebras. In W. Hodges, M. Hyland, C. Steinhorn, and J. Truss, editors, Logic: from foundations to applications, pages 187-213. Clarendon Press, Oxford, 1996.

[32] Dick de Jongh and Frank Veltman. Provability logics for relative interpretability. In Petio Petrov Petkov, editor, Mathematical Logic, pages 31-42. Springer US, Boston, MA, 1990.

[33] Valeria de Paiva, Rajeev Goré, and Michael Mendler. Editorial: Modalities in constructive logics and type theories. Journal of Logic and Computation, 14(4):439-446, 2004.

[34] Valeria de Paiva and Eike Ritter. Basic constructive modality. In Jean-Yves Beziau and Marcelo Esteban Coniglio, editors, Logic Without Frontiers- Festschrift for Walter Alexandre Carnielli on the occasion of his 60th birthday, pages 411-428. College Publications, 2011.

[35] M. A. E. Dummett and E. J. Lemmon. Modal logics between S4 and S5. Zeitschrift für Mathematische Logik und Grundlagen der Mathematik, 5:250-264, 1959.

[36] L. L. Esakia. Topological Kripke models. Soviet Mathematics Doklady, 15:147-151, 1974.

[37] L. L. Esakia. On varieties of Grzegorczyk algebras. In Studies in non-classical logics and set theory, pages 257-287, Moscow, 1979. Nauka Press. In Russian.

[38] L. L. Esakia. Heyting Algebras. Trends in Logic. Springer, Springer, 2019. Translated by A. Evseev.

[39] Matt Fairtlough and Michael Mendler. Propositional lax logic. Inform. and Comput., 137(1):1-33, 1997.

[40] Frederic B. Fitch. Intuitionistic modal logic with quantifiers. Portugaliae mathematica, 7(2):113$118,1948$.

[41] Josep Maria Font. Beyond Rasiowa's algebraic approach to non-classical logics. Stud. Logica, 82(2):179-209, 2006.

[42] Josep Maria Font, Ramon Jansana, and Don Pigozzi. A survey of abstract algebraic logic. Stud. Logica, 74(1-2):13-97, 2003.

[43] Deepak Garg and Frank Pfenning. Non-interference in constructive authorization logic. In Proc. of CSFW, pages 283-296. IEEE Computer Society, 2006.

[44] Dimiter Todorov Georgiev. Algorithmic Methods for Non-Classical Logics. PhD thesis, Sofia University, 2017.

[45] K. Gödel. Einde Interpretation des intuitionistischen Aussagenkalkuls. Ergebnisse eines mathematischen Kolloquiums, 6:39-40, 1933.

[46] Robert I. Goldblatt. Cover semantics for quantified lax logic. J. Log. Comput., pages 1035-1063, 2010.

[47] J. de Groot and D. Pattinson. Modal intuitionistic logics as dialgebraic logics. In Proc. LICS'20, pages 355-369, New York, 2020. Association for Computing Machinery.

[48] John Hughes. Generalising monads to arrows. Sci. Comput. Programming, 37(1-3):67-111, 2000.

[49] John Hughes. Programming with arrows. In Varmo Vene and Tarmo Uustalu, editors, Revised 
Lectures, AFP 2004, volume 3622 of Lecture Notes in Computer Science, pages 73-129. Springer, 2004.

[50] R. Iemhoff. A modal analysis of some principles of the provability logic of Heyting Arithmetic. In Proceedings of AiML'98, volume 2, Uppsala, 2001.

[51] Rosalie Iemhoff. Provability Logic and Admissible Rules. PhD thesis, University of Amsterdam, 2001.

[52] Rosalie Iemhoff. Preservativity logic: An analogue of interpretability logic for constructive theories. Math. Log. Quart., 49(3):230-249, 2003.

[53] Rosalie Iemhoff, Dick De Jongh, and Chunlai Zhou. Properties of intuitionistic provability and preservativity logics. Log. J. IGPL, 13(6):615-636, 2005.

[54] Guilhem Jaber, Nicolas Tabareau, and Matthieu Sozeau. Extending type theory with forcing. In Proc. of LiCS, pages 395-404. IEEE, 2012.

[55] Bart Jacobs, Chris Heunen, and Ichiro Hasuo. Categorical semantics for arrows. J. Funct. Programming, 19(3-4):403-438, 2009.

[56] Ralf Jung, Robbert Krebbers, Jacques-Henri Jourdan, Ales Bizjak, Lars Birkedal, and Derek Dreyer. Iris from the ground up: A modular foundation for higher-order concurrent separation logic. J. Funct. Program., 28:e20, 2018.

[57] G. A. Kavvos. Dual-Context Calculi for Modal Logic. Logical Methods in Computer Science, Volume 16, Issue 3, August 2020.

[58] Satoshi Kobayashi. Monad as modality. Theoretical Computer Science, 175(1):29 - 74, 1997.

[59] Neelakantan R. Krishnaswami and Nick Benton. A semantic model for graphical user interfaces. In Manuel M. T. Chakravarty, Zhenjiang Hu, and Olivier Danvy, editors, Proc. of ICFP, pages 45-57. ACM SIGPLAN, ACM, 2011.

[60] Neelakantan R. Krishnaswami and Nick Benton. Ultrametric semantics of reactive programs. In Proc. of LiCS, pages 257-266. IEEE, 2011.

[61] C. Kupke, A. Kurz, and Y. Venema. Stone coalgebras. Theoretical Computer Science, 327(1):109134, 2004. Selected Papers of CMCS '03.

[62] Alexander Kurz and Alessandra Palmigiano. Epistemic updates on algebras. LMCS, 9(4), 2013.

[63] C. I. Lewis. The matrix algebra for implications. The Journal of Philosophy, Psychology and Scientific Methods, 11(22):589-600, 1914.

[64] C. I. Lewis. Strict implication-an emendation. The Journal of Philosophy, Psychology and Scientific Methods, 17(11):300-302, 1920.

[65] C. I. Lewis. Alternative systems of logic. The Monist, 42(4):481-507, 1932.

[66] C.I. Lewis. A Survey of Symbolic Logic. University of California Press, 1918.

[67] C.I. Lewis. Logic and pragmatism. In G. P. Adams and W. P. Montague, editors, Contemporary American Philosophy: Personal Statements, volume 2 of Library of philosophy, ed. by J. $H$. Muirhead. G. Allen \& Unwin Limited, 1930.

[68] C.I. Lewis and C.H. Langford. Symbolic Logic. Dover, 1932.

[69] Sam Lindley, Philip Wadler, and Jeremy Yallop. The arrow calculus. J. Funct. Programming, 20:51-69, 12010.

[70] Sam Lindley, Philip Wadler, and Jeremy Yallop. Idioms are oblivious, arrows are meticulous, monads are promiscuous. ENTCS, 229(5):97-117, 2011. Proc. of MSFP.

[71] T. Litak and A. Visser. Lewis meets Brouwer: Constructive strict implication. Indagationes Mathematicae, 29(1):36-90, 2018.

[72] Tadeusz Litak. Constructive modalities with provability smack. In Guram Bezhanishvili, editor, Leo Esakia on duality in modal and intuitionistic logics, volume 4 of Outstanding Contributions to Logic, pages 179-208. Springer, 2014.

[73] Tadeusz Litak, Miriam Polzer, and Ulrich Rabenstein. Negative Translations and Normal Modal- 
ity. In Dale Miller, editor, Proc. of FSCD, volume 84 of LIPIcs, pages 27:1-27:18, Dagstuhl, Germany, 2017. Schloss Dagstuhl-Leibniz-Zentrum fuer Informatik.

[74] Tadeusz Litak and Albert Visser. Lewisian fixed points I: two incomparable constructions. CoRR, abs/1905.09450, 2019.

[75] L. L. Maksimova and V. V. Rybakov. A lattice of normal modal logics. Algebra and Logic, $13: 105-122,1974$.

[76] Edwin D. Mares. Relevant Logic: A Philosophical Interpretation. Cambridge University Press, 2004.

[77] Conor McBride and Ross Paterson. Applicative programming with effects. J. Funct. Programming, 18(1):1-13, 2008.

[78] J. C. C. McKinsey and A. Tarski. Some theorems about the sentential calculi of lewis and heyting. The Journal of Symbolic Logic, 13(1):1-15, 1948.

[79] Luka Mikec, Fedor Pakhomov, and Mladen Vuković. Complexity of the interpretability logic IL. Log. J. IGPL, 27(1):1-7, 2019.

[80] Luka Mikec, Tin Perkov, and Mladen Vuković. Decidability of interpretability logics ILM I $_{0}$ and ILW*. Logic Journal of the IGPL, 25(5):758-772, 072017.

[81] Stefan Milius and Tadeusz Litak. Guard your daggers and traces: Properties of guarded (co)recursion. Fundamenta Informaticae, 150:407-449, 2017. special issue FiCS'13 edited by David Baelde, Arnaud Carayol, Ralph Matthes and Igor Walukiewicz.

[82] Eugenio Moggi. Notions of computation and monads. Inform. and Comput., 93:55-92, July 1991.

[83] P.J. Morandi. Dualities in lattice theory, $2005 . \quad$ Available at http://sierra.nmsu.edu/morandi/notes/Duality.pdf.

[84] Hiroshi Nakano. A modality for recursion. In Proc. of LiCS, pages 255-266. IEEE, 2000.

[85] Hiroshi Nakano. Fixed-point logic with the approximation modality and its Kripke completeness. In Naoki Kobayashi and Benjamin C. Pierce, editors, Proc. of TACS, volume 2215 of LNCS, pages 165-182. Springer, 2001.

[86] Aleksandar Nanevski and Frank Pfenning. Staged computation with names and necessity. $J$. Funct. Program., 15(5):893-939, 2005.

[87] H. Ono. On some intuitionistic modal logics. Publications of the Research Institute for Mathematical Sciences, 13:687-722, 1977.

[88] William Tuthill Parry. In memoriam: Clarence Irving Lewis (1883-1964). Notre Dame J. Formal Logic, 11(2):129-140, 041970.

[89] Tin Perkov and Mladen Vuković. Filtrations of generalized Veltman models. Mathematical Logic Quarterly, 62(4-5):412-419, 2016.

[90] Carlo Proietti. Intuitionistic epistemic logic, kripke models and fitch's paradox. J. Philos. Log., 41(5):877-900, 2012.

[91] H. Rasiowa. An Algebraic Approach to Non-classical Logics. North Holland, 1974.

[92] Daniel Rogozin. Categorical and algebraic aspects of the intuitionistic modal logic IEL ${ }^{-}$and its predicate extensions. Journal of Logic and Computation, 12 2020. exaa082.

[93] G. Sambin. An effective fixed-point theorem in intuitionistic diagonalizable algebras. Stud. Logica, 35:345-361, 1976.

[94] G Fischer Servi. On modal logics with an intuitionistic base. Studia Logica, 36:141-149, 1977.

[95] V. B. Shehtman. Kripke type semantics for propositional modal logics with intuitionistic base. In V. A. Smirnov, editor, Modal and Tense Logics, pages 108-112. Institute of Philosophy, USSR Academy of Sciences, 199. In Russian.

[96] Alex K. Simpson. The Proof Theory and Semantics of Intuitionistic Modal Logic. PhD thesis, University of Edinburgh, 1994.

[97] Robert M. Solovay. Provability interpretations of modal logic. Israel J. Math., 25:287-304, 1976. 
[98] Vladimir Sotirov. Modal theories with intuitionistic logic. In Mathematical Logic, Proc. Conf. Math. Logic Dedicated to the Memory of A. A. Markov (1903 - 1979), Sofia, September 22 - 23, 1980, pages 139-171, 1984.

[99] J. F. A. K. van Benthem. Modal frame correspondences and fixed-points. Studia Logica, 83(13):133-155, 2006.

[100] A. Visser. Propositional combinations of $\Sigma$-sentences in Heyting's Arithmetic. Logic Group Preprint Series 117. Faculty of Humanities, Philosophy, Utrecht University, Janskerkhof 13, 3512 BL Utrecht, 1994.

[101] A. Visser. Substitutions of $\Sigma_{1}^{0}$-sentences: explorations between intuitionistic propositional logic and intuitionistic arithmetic. Annals of Pure and Applied Logic, 114:227-271, 2002.

[102] A. Visser. Closed fragments of provability logics of constructive theories. Journal of Symbolic Logic, 73(3):1081-1096, 2008.

[103] Albert Visser. On the completeness principle: A study of provability in Heyting Arithmetic and extensions. Ann. Math. Logic, 22(3):263-295, 1982.

[104] Albert Visser. Löb's logic meets the $\mu$-calculus. In Aart Middeldorp, Vincent van Oostrom, Femke van Raamsdonk, and Roel C. de Vrijer, editors, Processes, Terms and Cycles: Steps on the Road to Infinity, Essays Dedicated to Jan Willem Klop, on the Occasion of His 60th Birthday, volume 3838 of $L N C S$, pages 14-25. Springer, 2005.

[105] Y. Weiss. Basic intuitionistic conditional logic. Journal of Philosophical Logic, 48(3):447-469, 2019.

[106] Y. Weiss. Frontiers of conditional logic. PhD thesis, City University of New York, 2019.

[107] Timothy Williamson. On intuitionistic modal epistemic logic. J. Philos. Log., 21(1):63-89, 1992.

[108] F. Wolter and M. Zakharyaschev. On the relation between intuitionistic and classical modal logics. Algebra and Logic, 36:121-155, 1997.

[109] F. Wolter and M. Zakharyaschev. Intuitionistic modal logics as fragments of classical bimodal logics. In E. Orlowska, editor, Logic at Work, Essays in honour of Helena Rasiowa, pages 168186. Springer-Verlag, 1998.

[110] F. Wolter and M. Zakharyaschev. Intuitionistic modal logic. In A. Cantini, E. Casari, and P. Minari, editors, Logic and Foundations of Mathematics: Selected Contributed Papers of the Tenth International Congress of Logic, Methodology and Philosophy of Science, pages 227-238, Dordrecht, 1999. Springer Netherlands. 


\section{A Appendix}

Here we collect details omitted from the main body of the text. Appendix A.1 elaborates on Remark 3.13. Appendices A.2, A.3, A.4, A.5 and A.6 give proofs of Lem. 3.19, 4.13, 4.18, 4.19 and Theorem 5.4. These are given their own appendix because they require additional definitions and lemmas.

\section{A.1 Descriptive -3 -frames as spaces}

We work out the details of Remark 3.13. Recall that a Stone space is a zero-dimensional compact Hausdorff space. An Esakia space [36] is a tuple $(X, \preceq, \tau)$ consisting of a Stone space $(X, \tau)$ and a relation $\preceq$ on $X$ that satisfies

- $\uparrow_{\preceq} x=\{y \in X \mid x \preceq y\}$ is closed in $(X, \tau)$ for each $x \in X$;

- $\downarrow_{\preceq} a=\{y \in X \mid y \preceq x$ for some $x \in a\}$ is clopen in $(X, \tau)$ for all clopen $a \in \tau$.

Together with bounded continuous morphisms, Esakia spaces for the category Esa. It is well known that Esa is isomorphic to the category of descriptive intuitionistic Kripke frames and general intuitionistic Kripke frame morphisms [36,38].

Piggy-backing on this, we develop a topological perspective of descriptive -3-frames.

A.1 Definition. A strict implication space is a tuple $(X, \preceq, \sqsubset, \tau)$ such that $(X, \preceq, \tau)$ is an Esakia space and $\sqsubset$ is a binary relation on $X$ such that

$1 x \preceq y \sqsubset z$ implies $x \sqsubset z$ for all $x, y, z \in X$;

$2 \uparrow_{\sqsubset} x=\{y \in X \mid x \sqsubset y\}$ is closed in $(X, \tau)$ for all $x \in X$;

$3 \downarrow_{\sqsubset} a=\{x \in X \mid x \sqsubset y$ for some $y \in a\}$ is clopen for every clopen $a \subseteq X$.

These constitute the category SIS, whose morphisms are continuous morphisms that are bounded with respect to both relations.

A.2 Theorem. We have D-Frm $\cong$ SIS.

Proof. Let $(X, \preceq, \sqsubset, P)$ be a descriptive - -frame and write $\tau_{P}$ for the topology on $X$ generated by the subbase $P$. Since $(X, \preceq, P)$ is a descriptive intuitionistic Kripke frame we know that $\left(X, \preceq, \tau_{P}\right)$ is an Esakia space. Furthermore, $\left(\mathrm{S}_{1}\right)$ follows from the fact that $(X, \preceq, \sqsubset)$ is a $-3-$ frame and $\left(\mathrm{S}_{2}\right)$ follows from $\sqsubset$-refinedness. For $\left(\mathrm{S}_{3}\right)$, let $a$ be a clopen subset of $X$. Then $a=\bigcup_{i=1}^{n} b_{i} \cap-c_{i}$, where $b_{i}, c_{i} \in P$, and we have

$$
\begin{aligned}
\downarrow_{\sqsubset} a & =\bigcup \downarrow_{\sqsubset}\left(b_{i} \cap-c_{i}\right) \\
& =\bigcup-\left(b_{i} \underline{-} \underline{c} c_{i}\right)
\end{aligned}
$$

which is in $-P$.

Conversely, for a strict implication space $(X, \preceq, \sqsubset, \tau)$ let $P$ be the collection of clopen upsets of $(X, \tau)$. Then $(X, \preceq, P)$ is a descriptive intuitionistic Kripke frame, $(X, \preceq, \sqsubset)$ is a - -frame because of $\left(\mathrm{S}_{1}\right)$, $\sqsubset$-refinedness follows from $\left(\mathrm{S}_{2}\right)$ and an argument that resembles the proof of Proposition, and closure of $P$ under $\underline{3}$ follows from the fact that $a \underline{3} b=X \backslash \downarrow_{\sqsubset}(a \cap-b)$ is in $P$ as a consequence of $\left(\mathrm{S}_{3}\right)$.

It is obvious that these two transformations define a bijection on objects. The isomorphism on morphisms is trivial. 


\section{A.2 Proof of Lemma 3.19}

For the proof of Lem. 3.19 we make use of the following auxiliary lemma.

A.3 Lemma. Let $(A,-3)$ be an $\mathrm{HL}$-algebra. Then for all $a, b, c \in A$ we have

1. $a-b \leq(a \wedge c)-3 b$ (antitone in first argument)

2. $a-3 b \leq(a \wedge c)-3(b \wedge c)$

Proof. It follows from C2 and absorption that

$$
(a \multimap b) \wedge((a \wedge c) \dashv b)=(a \vee(a \wedge c))-3 b=a \dashv b
$$

and this entails the first item. The second item follows from [71, Lem. 4.1(a)].

Proof of Lemma 3.19. We prove two inclusions. Suppose $\mathfrak{p} \in \widetilde{a-3 b}$, so that $a-3 b \in \mathfrak{p}$. If $\mathfrak{p} \sqsubset \mathfrak{q}$ and $\mathfrak{q} \in \widetilde{a}$, then $a \in \mathfrak{q}$. By definition of $\sqsubset$ this implies $b \in \mathfrak{q}$, hence $\mathfrak{q} \in \widetilde{b}$. Therefore $\mathfrak{p} \in \widetilde{a} \_\widetilde{b}$.

For the converse we need to work harder. Suppose $\mathfrak{p} \notin \widetilde{a-3 b}$. We aim to find a prime filter $\mathfrak{q} \in p f A$ such that:

(1) $\mathfrak{p} \sqsubset \mathfrak{q}$;

(2) $a \in \mathfrak{q}$;

(3) $b \notin \mathfrak{q}$.

By definition of $\underline{3}$ this entails $\mathfrak{p} \notin \widetilde{a} \underline{\underline{3}} \widetilde{b}$.

We aim to construct the desired prime filter $\mathfrak{q}$ using the prime filter lemma. To this end, let $F=\{c \in A \mid a \preceq c\}$ and $I=\{d \in A \mid d-3 b \in \mathfrak{p}\}$. Trivially $F$ is a filter, and it follows from C2 that $I$ is an ideal. In particular, we have $a \in F$ and $b \in I$. Moreover, $F \cap I=\emptyset$. To see this, suppose $x \in F \cap I$. Then $a \preceq x$ and $x-b \in p$, so by Lem. A.3(1) $x-b \preceq a \preccurlyeq b$ and since $p$ is a filter $a-3 b \in \mathfrak{p}$. A contradiction.

Thus we can invoke the prime filter lemma to obtain a prime filter $\mathfrak{q}$ containing $F$ and disjoint from $I$. But more is true: if we have a look at the proof of the prime filter lemma (see e.g. [83, Lem. 1.4]) we see that $\mathfrak{q}$ is a maximal element with the property that it is a filter containing $F$ disjoint from $I$. That is, it is a maximal element in the set

$$
\mathscr{P}=\{\mathfrak{s} \in \text { fil } A \mid F \subseteq \mathfrak{s} \text { and } \mathfrak{s} \cap I=\emptyset\},
$$

which is ordered by inclusion.

Items ( $(2))$ and $((3))$ are already satisfied We will now prove that $\mathfrak{q}$ is such that $\mathfrak{p} \sqsubset \mathfrak{q}$. Suppose towards a contradiction that $\mathfrak{p} \not \subset \mathfrak{q}$. Then we can find $c, d \in A$ such that $c-3 d \in \mathfrak{p}$ and $c \in \mathfrak{q}$ and $d \notin \mathfrak{q}$. Let $\mathfrak{q}_{d}$ be the filter generated by $\mathfrak{q} \cup\{d\}$. It is easy to check that

$$
\mathfrak{q}_{d}=\{c \wedge e \mid c \in \mathfrak{q}, d \preceq e\} .
$$

Since $\mathfrak{q}_{d}$ properly contains $\mathfrak{q}$ and $\mathfrak{q}$ is maximal in $\mathscr{P}$, we must have $\mathfrak{q}_{d} \notin \mathscr{P}$. This implies $\mathfrak{q}_{d} \cap I \neq \emptyset$, and therefore we can find $e \in \mathfrak{q}$ such that $e \wedge d \in I$. By definition of $I$ this means $(e \wedge d)-3 b \in \mathfrak{p}$. By assumption $c-3 d \in \mathfrak{p}$ and so it follows from Lem. A.3(2) that $(e \wedge c) \multimap(e \wedge d) \in \mathfrak{p}$. By C3,

$$
((e \wedge c) \dashv(e \wedge d)) \wedge((e \wedge d) \neg b) \preceq(e \wedge c) \dashv b
$$


and since $\mathfrak{p}$ is a filter this implies $(e \wedge c)-3 b \in \mathfrak{p}$. By definition of $I$ we now have $e \wedge c \in I$. But we chose both $e$ and $c$ from $\mathfrak{q}$, and since $\mathfrak{q}$ is a filter this implies $e \wedge c \in \mathfrak{q}$. This is a contradiction with the fact that $\mathfrak{q}$ and $I$ are disjoint, hence the assumption that $\mathfrak{p} \not \subset \mathfrak{q}$ must be false. We conclude that $\mathfrak{p} \sqsubset \mathfrak{q}$. This completes the proof of the lemma.

Proof of Proposition 3.20. We know from intuitionistic logic that $(p f A, \subseteq, \widetilde{A})$ is a descriptive intuitionistic Kripke frame. To show that $(p f A, \subseteq, \sqsubset)$ is a -3 -frame, suppose $\mathfrak{p}^{\prime} \subseteq \mathfrak{p} \sqsubset \mathfrak{q}$. Then $a \dashv b \in \mathfrak{p}^{\prime}$ implies $a \dashv b \in \mathfrak{p}$, so whenever $a \in \mathfrak{q}$ we also have $b \in \mathfrak{q}$. So $\mathfrak{p}^{\prime} \sqsubset \mathfrak{q}$. Lemma 3.19 entails that $\widetilde{A}$ is closed under $\underline{-}$ because

$$
\widetilde{a} \underline{3} \widetilde{b}=\widetilde{a-3 b} \in \widetilde{A} \text {. }
$$

Finally, it follows from the construction of $\sqsubset$ that $\mathcal{A}_{*}$ is $\sqsubset$-refined, and since it is based on a descriptive intuitionistic Kripke frame, $\mathcal{A}_{*}$ is descriptive.

Proof of Lemma 3.21. This follows from the fact that the maps involved piggy-back on those from the duality between Heyting algebras and descriptive intuitionistic Kripke frames, which are natural.

Proof of Theorem 3.22. It suffices to prove that we have natural isomorphisms $i d_{\mathrm{HLAs}} \cong\left((\cdot)_{*}\right)^{*}$ and $i d_{\mathrm{D}-\mathrm{Frm}} \cong\left((\cdot)^{*}\right)_{*}$. The former was proved in Lem. 3.21, so we focus on the latter.

Let $\mathfrak{G}=(X, \preceq, \sqsubset, P)$ be a descriptive frame. We already know that $\widehat{(\cdot)}: \mathfrak{G} \rightarrow\left(\mathfrak{G}^{*}\right)_{*}$ given by $\widehat{x}=\{a \in P \mid x \in a\}$ is a natural isomorphism between the underlying descriptive intuitionistic Kripke frames $[23, \S 8.4]$. So it suffices to prove that $\widehat{(\cdot)}$ is a 3 -frame morphism. Since $\widehat{(\cdot)}$ is a bijection, it is enough to prove that $x \sqsubset y$ if and only if $\widehat{x} \sqsubset \widehat{y}$. But this follows immediately from the definition of our functors:

$$
\begin{aligned}
& x \sqsubset y \text { iff } \quad \forall a, b \in P[x \in a \underline{-3} b \text { and } y \in a \text { imply } y \in b] \\
& \text { iff } \forall a, b \in P[a \underline{-3} b \in \widehat{x} \text { and } a \in \widehat{y} \text { imply } b \in \widehat{y}] \\
& \text { iff } \widehat{x} \sqsubset \widehat{y} .
\end{aligned}
$$

This proves the theorem.

\section{A.3 Proof of Lemma 4.13}

The proof of Lem. 4.13 follows from the following two lemmas. Before each of these we introduce the relevant notion of the translation of a valuation.

Let $\mathfrak{F}=\left(X, R_{\mathrm{i}}, R_{\mathrm{m}}, P\right)$ be a general S4K-frame. If $V: \mathrm{At} \rightarrow P$ is a valuation for it, then we define the valuation $\widehat{\rho} V:$ At $\rightarrow \widehat{\rho} P$ for $\widehat{\rho} \mathfrak{F}=\left(\lfloor X\rfloor,\left\lfloor R_{\mathrm{i}}\right\rfloor,\left\lfloor R_{\mathrm{m}}^{*}\right\rfloor, \widehat{\rho} P\right)$ by

$$
\widehat{\rho} V(p):=\lfloor[i] V(p)\rfloor=[\mathrm{i}](\bigcup\lfloor[i] V(p)\rfloor) .
$$

A.4 Lemma. Let $\mathfrak{F}=\left(X, R_{\mathrm{i}}, R_{\mathrm{m}}, P\right)$ be a general $\mathrm{S} 4 \mathrm{~K}$-frame and $V$ a valuation for $\mathfrak{F}$. Then we have

$$
(\mathfrak{F}, V), x \Vdash t(\varphi) \quad \text { iff } \quad(\widehat{\rho} \mathfrak{F}, \widehat{\rho} V),\lfloor x\rfloor \Vdash \varphi
$$

for all $x \in X$ and $\varphi \in \mathcal{L}_{-3}$. 
Proof. By induction on the structure of $\varphi$. The cases $\top$ and $\perp$ are obvious.

$\varphi=p \in \mathrm{At}$ We have

$$
\begin{array}{rlll}
(\mathfrak{F}, V), x \Vdash t(p)=\square_{\mathrm{i}} p \quad \text { iff } & R_{\mathrm{i}}[x] \subseteq V(p) \\
& \text { iff } & x \in[\mathrm{i}] V(p) \\
\text { iff } & \lfloor x\rfloor \in\lfloor[i] V(p)\rfloor=\widehat{\rho} V(p) \\
& \text { iff } & (\widehat{\rho} \mathfrak{F}, \widehat{\rho} V),\lfloor x\rfloor \Vdash p
\end{array}
$$

The right-to-left direction from the third "iff" follows from the fact that $x \sim x^{\prime}$ implies $x^{\prime} \in$ [i] $V(p)$.

$\varphi=\varphi_{1} \wedge \varphi_{2}$ We have

$$
\begin{aligned}
&(\mathfrak{F}, V), x \Vdash t\left(\varphi_{1} \wedge \varphi_{2}\right)=\square_{\mathrm{i}}\left(t\left(\varphi_{1}\right) \wedge t\left(\varphi_{2}\right)\right) \\
& \text { iff } \quad x R_{\mathrm{i}} y \Rightarrow y \Vdash t\left(\varphi_{1}\right) \text { and } y \Vdash t\left(\varphi_{2}\right) \\
& \text { iff } \quad\lfloor x\rfloor\left\lfloor R_{\mathrm{i}}\right\rfloor\lfloor y\rfloor \Rightarrow\lfloor y\rfloor \Vdash \varphi_{1} \text { and }\lfloor y\rfloor \Vdash \varphi_{2} \\
& \text { iff } \quad\lfloor x\rfloor\left\lfloor R_{\mathrm{i}}\right\rfloor\lfloor y\rfloor \Rightarrow\lfloor y\rfloor \Vdash \varphi_{1} \wedge \varphi_{2} \\
& \text { iff } \quad(\widehat{\rho} \mathfrak{F}, \widehat{\rho} V),\lfloor x\rfloor \Vdash \varphi_{1} \wedge \varphi_{2}
\end{aligned}
$$

The second "iff" follows from the induction hypothesis and the definition of $\lfloor\cdot\rfloor$.

$\varphi=\varphi_{1} \vee \varphi_{2}$ Similar to the previous case.

$\varphi=\varphi_{1} \rightarrow \varphi_{2}$ Compute

$$
\begin{aligned}
&(\mathfrak{F}, V), x \Vdash t\left(\varphi_{1} \rightarrow \varphi_{2}\right)=\square_{\mathrm{i}}\left(t\left(\varphi_{1}\right) \rightarrow t\left(\varphi_{2}\right)\right) \\
& \text { iff } \quad x R_{\mathrm{i}} y \text { and } y \Vdash t\left(\varphi_{1}\right) \text { imply } y \Vdash t\left(\varphi_{2}\right) \\
& \text { iff } \quad\lfloor x\rfloor\left\lfloor R_{\mathrm{i}}\right\rfloor\lfloor\rfloor \text { and }\lfloor y\rfloor \Vdash \varphi_{1} \text { imply }\lfloor y\rfloor \Vdash \varphi_{2} \\
& \quad \text { iff } \quad(\widehat{\rho} \mathfrak{F}, \widehat{\rho} V),\lfloor x\rfloor \Vdash \varphi_{1} \rightarrow \varphi_{2}
\end{aligned}
$$

$\varphi=\varphi_{1}-\varphi_{2}$ First assume $(\mathfrak{F}, V), x \Vdash t\left(\varphi_{1}-3 \varphi_{2}\right)$. Compute

$$
\begin{aligned}
(\mathfrak{F}, V), x \Vdash t\left(\varphi_{1}-3 \varphi_{2}\right)=\square_{\mathrm{i}} \square_{\mathrm{m}}\left(t\left(\varphi_{1}\right) \rightarrow t\left(\varphi_{2}\right)\right) \\
\quad \text { iff } \quad x R_{\mathrm{i}} y \text { implies } y \Vdash \square_{\mathrm{m}}\left(t\left(\varphi_{1}\right) \rightarrow t\left(\varphi_{2}\right)\right) \\
\quad \text { iff } \quad x\left(R_{\mathrm{i}} \circ R_{\mathrm{m}}\right) z \text { implies } z \Vdash t\left(\varphi_{1}\right) \rightarrow t\left(\varphi_{2}\right) \\
\quad \text { iff } \quad x\left(R_{\mathrm{i}} \circ R_{\mathrm{m}}\right) z \text { and } z \Vdash t\left(\varphi_{1}\right) \text { imply } z \Vdash t\left(\varphi_{2}\right) \\
\text { iff } \quad x R_{\mathrm{m}}^{*} z \text { and } z \Vdash t\left(\varphi_{1}\right) \text { imply } z \Vdash t\left(\varphi_{2}\right)
\end{aligned}
$$

Now suppose $\lfloor x\rfloor\left\lfloor R_{\mathrm{m}}^{*}\right\rfloor\lfloor z\rfloor$ and $\lfloor z\rfloor \Vdash \varphi_{1}$. Then there exists $z^{\prime} \in X$ such that $z \sim z^{\prime}$ and $x R_{\mathrm{m}}^{*} z^{\prime}$. Since $\lfloor z\rfloor \Vdash \varphi_{1}$ by the induction hypothesis we have $z^{\prime} \Vdash t\left(\varphi_{1}\right)$, so by the derivation above $z^{\prime} \Vdash t\left(\varphi_{2}\right)$ and therefore $\lfloor z\rfloor=\left\lfloor z^{\prime}\right\rfloor \Vdash \varphi_{2}$. So $\lfloor x\rfloor \Vdash \varphi_{1}-3 \varphi_{2}$.

Conversely, suppose $\lfloor x\rfloor \Vdash \varphi_{1}-3 \varphi_{2}$. Then $x R_{\mathrm{m}}^{*} z$ implies $\lfloor x\rfloor\left\lfloor R_{\mathrm{m}}^{*}\right\rfloor\lfloor z\rfloor$ and the desired result follows from the induction hypothesis.

Let $\mathfrak{F}=\left(X, R_{\mathrm{i}}, R_{\mathrm{m}}, P\right)$ be a general $\mathrm{S} 4 \mathrm{~K}$-frame. Suppose $W:$ At $\rightarrow \widehat{\rho} P$ is a valuation for $\widehat{\rho} \mathfrak{F}$. Then since $W(p) \in \widehat{\rho} P$ it must be of the form [i] $\lfloor a\rfloor$ for some $a$ such that $\bigcup\lfloor a\rfloor \in P$. We pick such $a$ and call it $W^{\prime}(p)$. 
A.5 Lemma. Let $\mathfrak{F}=\left(X, R_{\mathrm{i}}, R_{\mathrm{m}}, P\right)$ be a general $\mathrm{S} 4 \mathrm{~K}$-frame and $W$ a valuation for $\widehat{\rho} \mathfrak{F}$. Then we have

$$
\left(\mathfrak{F}, W^{\prime}\right), x \Vdash t(\varphi) \quad \text { iff } \quad(\widehat{\rho} \mathfrak{F}, W),\lfloor x\rfloor \Vdash \varphi
$$

for all $x \in X$ and $\varphi \in \mathcal{L}_{3}$.

Proof. By induction on the structure of $\varphi$. The cases $T$ and $\perp$ are obvious.

$\varphi=p \in$ At If $\left(\mathfrak{F}, W^{\prime}\right), x \Vdash t(p)=\square_{\mathrm{i}} p$ then $x R_{\mathrm{i}} y$ implies $y \in W^{\prime}(p)$. By definition of $\lfloor\cdot\rfloor$ we have $x R_{\mathrm{i}} y$ iff $\lfloor x\rfloor\left\lfloor R_{\mathrm{i}}\right\rfloor\lfloor y\rfloor$ and by definition of $W^{\prime}(p)$ we have $y \in W^{\prime}(p)$ iff $\lfloor y\rfloor \in\left\lfloor W^{\prime}(p)\right\rfloor$. Therefore $\lfloor x\rfloor \in[i]\left\lfloor W^{\prime}(p)\right\rfloor=W(p)$, so that $(\widehat{\rho} \mathfrak{F}, W),\lfloor x\rfloor \Vdash p$.

Conversely, suppose $(\widehat{\rho} \mathfrak{F}, W),\lfloor x\rfloor \Vdash p$. Then $\lfloor x\rfloor \in[i]\left\lfloor W^{\prime}(p)\right\rfloor$, so $\lfloor x\rfloor\left\lfloor R_{\mathrm{i}}\right\rfloor\lfloor y\rfloor$ implies $\lfloor y\rfloor \in$ $\left\lfloor W^{\prime}(p)\right\rfloor$. Again as a consequence of the definitions this gives $x R_{\mathrm{i}} y$ implies $y \Vdash p$, so that $\left(\mathfrak{F}, W^{\prime}\right), x \Vdash \square_{\mathrm{i}} p=t(p)$.

All other cases are the same as in Lem. A.4.

\section{A.4 Proof of Lemma 4.18}

In this subsection we will denote the truth set of a formula $\varphi$ in a model $\mathfrak{M}$ with valuation $V$ by $V(\varphi)$, rather than $\llbracket \varphi \rrbracket^{\mathfrak{M}}$. This will make it easier to distinguish the several different valuations based on a single frame, used in Lem. A.6 and A.7. We first recall Lem. 24 from [108].

A.6 Lemma. Suppose $\mathfrak{M}=(\mathfrak{G}, V)$ is a model based on a partially ordered frame $\mathfrak{G}=(X, R, P)$ for $\mathrm{Grz}$ and $\Gamma$ is a finite set of formulae closed under subformulae. Then there is a model $\mathfrak{M}^{\prime}=\left(\widehat{\sigma} \widehat{\rho} \mathfrak{G}, V^{\prime}\right)$ based on the frame $\widehat{\sigma} \widehat{\rho} \mathfrak{G}=(X, R, \widehat{\sigma} \widehat{\rho} P)$ such that for every $\varphi \in \Gamma$,

$$
V(\square \varphi)=V^{\prime}(\square \varphi) .
$$

In this lemma $\widehat{\sigma}$ and $\widehat{\rho}$ denote the translations $\sigma$ and $\rho$ between general intuitionistic Kripke frames and general S4-frames.

Next, let us state the lemma we are trying to prove. It is an adaptation of [108, Lem. 25], where the assumption that the frame satisfies Mix is weakened to BHL. The difference with the proof in loc. cit. is that we add a proposition letter that mimics $\square \psi$ for each subformula $\psi$ of a given formula $\varphi$, on top of the proposition letters already added in the original proof. This allows us to obtain the same result with a weaker assumption.

A.7 Lemma. Let $\mathfrak{G}=\left(X, R_{\mathrm{i}}, R_{\mathrm{m}}, P\right)$ be an $\mathrm{S} 4 \mathrm{BHL}$-frame such that $R_{\mathrm{i}}$ is a partial order and $\mathfrak{G} \Vdash \mathrm{Grz}_{\mathrm{i}}$. Then for all $\varphi \in \mathcal{L}_{\mathrm{i}, \mathrm{m}}$ we have

$$
\mathfrak{G} \Vdash \varphi \quad \text { iff } \quad \widehat{\sigma} \widehat{\rho} \mathfrak{G} \Vdash \varphi .
$$

Proof. Since $\mathfrak{G}$ is an S4BHL-frame we have $\widehat{\sigma} \widehat{\rho} \mathfrak{G}=\left(X, R_{\mathrm{i}}, R_{\mathrm{m}}, \widehat{\sigma} \widehat{\rho} P\right)$, i.e., the relations $R_{\mathrm{i}}$ and $R_{\mathrm{m}}$ remain unchanged. The left-to-right implication in (4) follows from the fact that $\widehat{\sigma} \widehat{\rho} P \subseteq P$.

Conversely, suppose $\mathfrak{G}=\left(X, R_{\mathrm{i}}, R_{\mathrm{m}}, P\right)$ refutes a formula $\varphi$. Then there exists a valuation $V:$ At $\rightarrow P$ such that $\mathfrak{M}=(\mathfrak{G}, V)$ refutes $\varphi$. Using Lem. A.6, we will construct a valuation $W^{\prime}$ for $\widehat{\sigma} \widehat{\rho} \mathfrak{G}$ such that $\left(\widehat{\sigma} \widehat{\rho} \mathfrak{G}, W^{\prime}\right)$ refutes $\varphi$. 
For each subformula $\psi$ of $\varphi$ we fix a new proposition letter $q^{\psi}$. Now for $\psi \in \operatorname{Subf}(\varphi)$ we define $\psi^{q}$ recursively via

$$
\begin{aligned}
& \top^{q}=\top \\
& \perp^{q}=\top \\
& p^{q}=p \\
& \left(\chi_{1} \star \chi_{2}\right)^{q}=\chi_{1}^{q} \star \chi_{2}^{q} \\
& \text { for } p \in \mathrm{At} \\
& \left(\square_{\mathrm{i}} \chi\right)^{q}=\square_{\mathrm{i}} \chi^{q} \\
& \left(\square_{\mathrm{m}} \chi\right)^{q}=\square_{\mathrm{i}} q^{\chi} \\
& \text { for } \star \in\{\wedge, \vee, \rightarrow\}
\end{aligned}
$$

Let $\widehat{\Gamma}=\left\{\psi^{q} \mid \psi \in \operatorname{Subf}(\varphi)\right\} \cup\left\{q^{\psi} \mid \psi \in \operatorname{Subf}(\varphi)\right\}$. Observe that $\widehat{\Gamma}$ is closed under subformulae. Define a valuation $W$ of the extended language by

$$
\begin{aligned}
W(p) & =V(p) & & \text { for } p \in \operatorname{Subf}(\varphi) \\
W\left(q^{\psi}\right) & =V\left(\square_{\mathrm{m}} \psi\right) & & \text { for } \psi \in \operatorname{Subf}(\varphi)
\end{aligned}
$$

Then for $\psi \in \operatorname{Subf}(\varphi)$ we have

$$
V\left(\square_{\mathrm{m}} \psi\right)=W\left(\square_{\mathrm{m}} \psi\right)=W\left(q^{\psi}\right)
$$

and

$$
V(\psi)=W(\psi)=W\left(\psi^{q}\right) .
$$

By Lem. A.6 there exists a valuation $W^{\prime}$ for $\widehat{\sigma} \widehat{\rho} \mathfrak{G}$ such that

$$
W^{\prime}\left(\square_{\mathbf{i}} \psi\right)=W\left(\square_{\mathbf{i}} \psi\right)
$$

for all $\psi \in \widehat{\Gamma}$. One can now prove that

$$
W^{\prime}\left(\psi^{q}\right)=W^{\prime}(\psi)
$$

for all $\psi \in \operatorname{Subf}(\varphi)$, by induction on the structure of $\psi$. The only non-trivial case is for $\square \psi \in \operatorname{Subf}(\varphi)$. Then we have

$$
\begin{aligned}
W^{\prime}\left(\left(\square_{\mathrm{m}} \psi\right)^{q}\right) & =W^{\prime}\left(\square_{\mathrm{i}} q^{\psi}\right) \\
& =W\left(\square_{\mathrm{i}} q^{\psi}\right) \\
& =W\left(\left(\square_{\mathrm{m}} \psi\right)^{q}\right) \\
& =W\left(\square_{\mathrm{m}} \psi\right) \\
& =W\left(\square_{\mathrm{i}} \square_{\mathrm{m}} \psi\right) \\
& =W\left(\square_{\mathrm{i}} \square_{\mathrm{m}} \psi^{q}\right) \\
& =W^{\prime}\left(\square_{\mathrm{i}} \square_{\mathrm{m}} \psi^{q}\right) \\
& =W^{\prime}\left(\square_{\mathrm{i}} \square_{\mathrm{m}} \psi\right) \\
& =W^{\prime}\left(\square_{\mathrm{m}} \psi\right)
\end{aligned}
$$

(By BHL)

This proves the claim in (8). 
Since every S4-model $\mathfrak{N}$ satisfies $\mathfrak{N} \Vdash \varphi$ iff $\mathfrak{N} \Vdash \square_{i} \varphi$, it suffices to prove that $W^{\prime}\left(\square_{i} \varphi\right)=$ $V\left(\square_{i} \varphi\right)$. We have

$$
\begin{aligned}
W^{\prime}\left(\square_{\mathrm{i}} \varphi\right) & =W^{\prime}\left(\left(\square_{\mathrm{i}}\right) \varphi^{q}\right) \\
& =W^{\prime}\left(\square_{\mathrm{i}} \varphi^{q}\right) \\
& =W\left(\square_{\mathrm{i}} \varphi^{q}\right) \\
& =V\left(\square_{\mathrm{i}} \varphi\right)
\end{aligned}
$$

so that $\left(\widehat{\sigma} \widehat{\rho} \mathfrak{G}, W^{\prime}\right)$ refutes $\square_{\mathrm{i}} \varphi$ (hence $\varphi$ ), as desired.

\section{A.5 Unravelling general S4K-frames}

We define the unravelling ur $\mathfrak{X}$ of a general S4K-frame $\mathfrak{X}$ and prove some properties. We will use a combination of unravelling techniques from Theorems 2.19 and 3.18 of [23].

Let $\mathfrak{X}=\left(X, R_{\mathrm{i}}, R_{\mathrm{m}}\right)$ be a S4K-frame and $x \in X$. We denote the unravelling of a pointed frame $(\mathfrak{X}, x)$ by ur $\mathfrak{X}=\left(X^{x}, R_{\mathrm{i}}^{x}, R_{\mathrm{m}}^{x}\right)$. For a construction, see [16, Prop. 2.15]. Here the elements of $X^{x}$ are finite paths over $R_{\mathrm{i}} \cup R_{\mathrm{m}}$ starting at $x$. Recall that we have a bounded morphism $f^{x}: \mathfrak{X}^{x} \rightarrow \mathfrak{X}$ given by sending $\left(x, x_{1}, \ldots, x_{n}\right) \in X^{x}$ to $x_{n} \in X$.

A.8 Definition. For an S4K-frame $\mathfrak{X}=\left(X, R_{\mathrm{i}}, R_{\mathrm{m}}\right)$ and $x \in X$ with unravelling ur $\mathfrak{X}=$ $\left(X^{x}, R_{\mathrm{i}}^{x}, R_{\mathrm{m}}^{x}\right)$, define $\bar{R}_{\mathrm{i}}^{x}$ to be the reflexive and transitive closure of $R_{\mathrm{i}}^{x}$ and define $\overline{u r} \mathfrak{X}=$ $\left(X^{x}, \bar{R}_{\mathrm{i}}^{x}, R_{\mathrm{m}}^{x}\right)$. We call $\overline{u r} \mathfrak{X}$ the partial unravelling of $\mathfrak{X}$.

Concretely, we have $\left(x, y_{1}, \ldots, y_{n}\right) \bar{R}_{\mathrm{i}}^{x}\left(x, y_{1}^{\prime}, \ldots, y_{m}^{\prime}\right)$ if either

- $m=n$ and $y_{i}=y_{i}^{\prime}$ for $1 \leq i \leq n$; or

- $m>n$ and $y_{i}=y_{i}^{\prime}$ for $1 \leq i \leq n$ and $y_{j}^{\prime} R_{\mathrm{i}} y_{j+1}^{\prime}$ for all $n \leq j<m$.

We call this the partial unravelling because it turns the pre-order $R_{\mathrm{i}}$ into a partial order $\bar{R}_{I}^{x}$.

A.9 Lemma. Let $(\mathfrak{X}, x)=\left(\left(X, R_{\mathrm{i}}, R_{\mathrm{m}}\right), x\right)$ be a pointed $\mathrm{S} 4 \mathrm{~K}$-frame with partial unravelling $\overline{u r}(\mathfrak{X}, x)=\left(X^{x}, \bar{R}_{\mathrm{i}}^{x}, R_{\mathrm{m}}^{x}\right)$. Then $\bar{R}_{\mathrm{i}}^{x}$ is a partial order.

Proof. We know that $\bar{R}_{\mathrm{i}}^{x}$ is reflexive and transitive by construction. Now suppose $\left(x, y_{1}, \ldots, y_{n}\right) \bar{R}_{\mathrm{i}}^{x}\left(x, y_{1}, \ldots, y_{m}\right)$ and $\left(x, y_{1}, \ldots, y_{m}\right) \bar{R}_{\mathrm{i}}^{x}\left(x, y_{1}, \ldots, y_{n}\right)$. Then we must have $n=m$ and by definition of $\bar{R}_{\mathrm{i}}^{x}$ we find $\left(x, y_{1}, \ldots, y_{n}\right)=\left(x, y_{1}, \ldots, y_{m}\right)$. So $\bar{R}_{\mathrm{i}}^{x}$ is also antisymmetric.

Since $R_{\mathrm{i}}$ is assumed to be a reflexive and transitive, we have:

A.10 Lemma. The function $f^{x}: X^{x} \rightarrow X$ is a bounded morphism from $\overline{u r} \mathfrak{X} \rightarrow \mathfrak{X}$.

Proof. We only need to verify that the additional relations that arise from reflexivity and transitivity do not compromise monotonicity of $f$. This follows from reflexivity and transitivty of $R_{\mathrm{i}}$.

Next we define the partial unravelling of a non-pointed frame.

A.11 Definition. Define the partial unravelling $\overline{u r} \mathfrak{X}$ of a (non-pointed) frame $\mathfrak{X}=\left(X, R_{\mathrm{i}}, R_{\mathrm{m}}\right)$ to be the coproduct (= disjoint union)

$$
\overline{u r} \mathfrak{X}=\coprod_{x \in X} \overline{u r}(\mathfrak{X}, x) .
$$

Write $\overline{u r} \mathfrak{X}=\left(X^{+}, \bar{R}_{\mathrm{i}}^{+}, R_{\mathrm{m}}^{+}\right)$. Then $X^{+}$is the disjoint union of the $X^{x}$, and $\bar{R}_{\mathrm{i}}^{+}$and $R_{\mathrm{m}}^{+}$are simply the unions of the relations $\bar{R}_{\mathrm{i}}^{x}$ and $\bar{R}_{M}^{x}$, respectively. 
By the property of a coproduct, there exists a bounded morphism $f=\coprod f^{x}: \overline{u r} \mathfrak{X} \rightarrow \mathfrak{X}$. Moreover, $f$ is onto because for each $x \in X$ we length-one sequence $(x)$ is in $X^{x}$, and $f^{x}(x)=$ $x \in X$. Besides, Lem. A.9 implies:

A.12 Lemma. If $R_{\mathrm{i}}$ is a pre-order on $X$, then $\bar{R}_{\mathrm{i}}^{+}$is a partial order on $X^{+}$.

The map $f: X^{+} \rightarrow X$ gives rise to a Boolean algebra homomorphism $f^{-1}: \mathcal{P} X \rightarrow \mathcal{P} X^{+}$. We claim that this moreover satisfies

$$
f^{-1}([\mathrm{i}] a)=[\mathrm{i}] f^{-1}(a), f^{-1}([\mathrm{~m}] a)=[\mathrm{m}] f^{-1}(a)
$$

for all $a \subseteq X$.

A.13 Lemma. Let $\mathfrak{X}=\left(X, R_{\mathrm{i}}, R_{\mathrm{m}}\right)$ be an S4K-frame with partial unravelling $\overline{\text { ur } \mathfrak{X}}=\left(X^{+}, \bar{R}_{\mathrm{i}}^{+}, R_{\mathrm{m}}^{+}\right)$. Write $(\wp X,[\mathrm{i}],[\mathrm{m}])$ and $\left(\wp X^{+},[\mathrm{i}]^{+},[\mathrm{m}]^{+}\right)$for the complex algebras of $\mathfrak{X}$ and $\overline{u r} \mathfrak{X}$, respectively. Then $f^{-1}: \wp X \rightarrow \wp X^{+}$defines a complex algebra homomorphism between them.

Proof. We know that $f^{-1}$ is a Boolean algebra homomorphism, so we only need

$$
f^{-1}([\mathrm{i}] a)=[\mathrm{i}]^{+} f^{-1}(a)
$$

and

$$
f^{-1}([\mathrm{~m}] a)=[\mathrm{m}]^{+} f^{-1}(a) .
$$

We start with the first equation. Suppose $\left(x, y_{1}, \ldots, y_{n}\right) \in f^{-1}([i] a)$. Then $y_{n} \in[i] a$. If $\left(x, y_{1}, \ldots, y_{n}\right) \bar{R}_{\mathrm{i}}^{+}\left(x, y_{1}^{\prime}, \ldots, y_{m}^{\prime}\right)$ then by definition of $\bar{R}_{\mathrm{i}}^{+}$and reflexivity and transitivity of $R_{\mathrm{i}}$ we have $y_{n} R_{\mathrm{i}} y_{m}^{\prime}$. Therefore $y_{m}^{\prime} \in a$, hence $f\left(x, y_{1}^{\prime}, \ldots, y_{m}^{\prime}\right) \in a$ so that $\left(x, y_{1}^{\prime}, \ldots, y_{m}^{\prime}\right) \in f^{-1}(a)$. This proves that $\left(x, y_{1}, \ldots, y_{n}\right) \in[\mathrm{i}]^{+} f^{-1}(a)$.

Conversely, suppose $\left(x, y_{1}, \ldots, y_{n}\right) \in[\mathrm{i}]^{+} f^{-1}(a)$. In order to prove that $\left(x, y_{1}, \ldots, y_{n}\right) \in$ $f^{-1}([\mathrm{i}] a)$, we need to show that $f\left(x, y_{1}, \ldots, y_{n}\right)=y_{n} \in[\mathrm{i}] a$. Let $y^{\prime} \in X$ be such that $y_{n} R_{\mathrm{i}} y^{\prime}$. $\left(x, y_{1}, \ldots, y_{n}\right) \bar{R}_{\mathrm{i}}^{+}\left(x, y_{1}, \ldots, y_{n}, y^{\prime}\right)$, so by assumption $\left(x, y_{1}, \ldots, y_{n}, y^{\prime}\right) \in f^{-1}(a)$ and hence $y^{\prime}=$ $f\left(x, y_{1}, \ldots, y_{n}, y^{\prime}\right) \in a$.

The computation for $[\mathrm{m}]$ are completely analogous.

Now let us define the general unravelling of a general S4K-frame.

A.14 Definition. Let $\mathfrak{G}=\left(X, \bar{R}_{\mathrm{i}}, R_{\mathrm{m}}, A\right)$ be a general S4K-frame. Denote by $\left(X^{+}, \bar{R}_{\mathrm{i}}^{+}, R_{\mathrm{m}}^{+}\right)$ be the partial unravelling of $\left(X, R_{\mathrm{i}}, R_{\mathrm{m}}\right)$ and let $f$ be the corresponding bounded morphism defined above. Then we define the general unravelling of $\mathfrak{G}$ to be the tuple

$$
\mathfrak{G}^{+}=\left(X^{+}, \bar{R}_{\mathrm{i}}^{+}, R_{\mathrm{m}}^{+}, A^{+}\right),
$$

where $A^{+}=\left\{f^{-1}(a) \subseteq X^{+} \mid a \in A\right\}$.

It follows from Lem. A.13 that the collection $A^{+}$does indeed define a complex algebra structure on $\left(X^{+}, \bar{R}_{\mathrm{i}}^{+}, R_{\mathrm{m}}^{+}\right)$.

Since $f$ is onto $f^{-1}$ is injective. Now conceive $f^{-1}$ as a function $A \rightarrow A^{+}$. Then by definition of $A^{+}$it is onto, so that $f^{-1}$ is a bijection. Moreover, it follows from Lem. A.13 that it is a complex algebra homomorphism. Since bijective homomorphisms in a variety of algebras are isomorphisms, it follows that $\mathfrak{X}^{*} \cong(\overline{\mathcal{u r}} \mathfrak{X})^{*}$. Therefore we have:

A.15 Proposition. Every classical bimodal logic $\Lambda$ containing $(\mathrm{S} 4 \otimes \mathrm{K})$ is characterised by a general S4K-frame $\mathfrak{G}=\left(X, R_{\mathrm{i}}, R_{\mathrm{m}}, P\right)$ such that $R_{\mathrm{i}}$ is a partial order.

Proof. Let $\mathfrak{G}$ be the descriptive frame which determines $\Lambda$. Then the partial unravelling $\overline{u r} \mathfrak{G}$ witnesses the claim. 


\section{A.6 Proof of Theorem 5.4}

The proof of Thm. 5.4 follows from the following two theorems, which consider subframe logics and $R_{\mathrm{m}}$-cofinal subframe logics, respectively.

A.16 Theorem. Suppose $\Theta$ is a canonical extension of $\mathrm{S} 4 \otimes \mathrm{K} 4$ containing $\mathrm{BHL}$ that is closed under forming subframes. Then:

1. $\Theta$ has the finite model property.

2. If moreover $\Theta$ contains the classical strength axiom

$$
\mathrm{S}_{\mathrm{c}} \square_{\mathrm{i}} p \rightarrow \square_{\mathrm{m}} p .
$$

then for any subframe logic $\Gamma \subseteq \mathcal{L}_{\mathrm{m}}$, the logic $\Theta \oplus \Gamma$ has the finite model property.

Proof of Theorem A.16. Let $\mathfrak{F}=\left(X, R_{\mathrm{i}}, R_{\mathrm{m}}, P\right)$ be a descriptive frame for $\Theta$ refuting a $\mathcal{L}_{\mathrm{i}, \mathrm{m}^{-}}$ formula $\varphi$ under valuation $V$. Then we will construct a finite subframe of $\mathfrak{F}$ that validates $\Theta$ and refutes $\varphi$. In order to prove this, we an adaptation of the proofs of Theorems 17 and 21 in [109].

We begin by constructing an inductive sequence $\left\{X_{i}\right\}_{i \in \omega}$ of subsets of $X$.

Base step. By [109, Lem. 14] we can pick a $R_{\mathrm{i}}$-maximal state $x_{0}$ where $\varphi$ is refuted. Let $\overline{X_{0}}=\left\{x_{0}\right\}$.

Odd inductive step. Suppose $n$ is even. For each $x^{\prime} \in X_{n}$ pick an $R_{\mathrm{i}}$-maximal witness for each

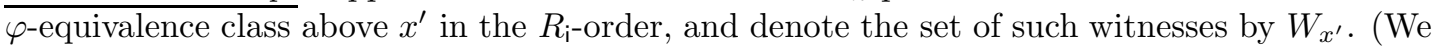
can find such witnesses by $[109$, Lem. 14].) Let

$$
X_{n+1}=X_{n} \cup \bigcup_{x^{\prime} \in X_{n}} W_{x^{\prime}} .
$$

The sets $W_{x^{\prime}}$ are finite because there are only finitely many $\varphi$-equivalence classes. Note also that in the presence of the strength axiom $\mathrm{S}_{\mathrm{a}}, R_{\mathrm{i}}$-maximal successors are also $R_{\mathrm{m}}$-maximal ones.

Even inductive step. Suppose $n$ is odd. For each $x^{\prime} \in X_{n+1}$, using [109, Lem. 14] pick an $R_{\mathrm{m}^{-}}$ maximal witness for each $\varphi$-equivalence class, and denote the set of such witnesses by $W_{x^{\prime}}$. Again, define $X_{n+1}=X_{n} \cup \bigcup_{x^{\prime} \in X_{n}} W_{x^{\prime}}$.

The set $X_{\omega}$. Define

$$
X_{\omega}:=\bigcup_{n \in \omega} X_{n} .
$$

Before turning this into a model, we shall argue that it is a finite subset of $X$. Our more general setting compared to [109] makes this more complicated, but not impossible.

For each $x \in X_{\omega}$, denote by $n(x)$ the smallest integer $n \in \omega$ such that $x \in X_{n}$. Define relations $R_{\mathrm{i}}^{\prime}$ and $R_{\mathrm{m}}^{\prime}$ on $X_{\omega}$ by

$$
\begin{array}{rll}
x R_{\mathfrak{i}}^{\prime} y & \text { if } & x R_{\mathrm{m}} y \text { and } n(y) \text { is odd and } y \in X_{n(y)-1} \\
x R_{\mathrm{m}}^{\prime} y & \text { if } & x R_{\mathrm{m}} y \text { and } n(y) \text { is even and } y \in X_{n(y)-1}
\end{array}
$$

Then $x R_{\mathrm{i}}^{\prime} y$ implies that $y$ is $R_{\mathrm{i}}$-maximal relative to $\sim_{\varphi}$, and similar for $R_{\mathrm{m}}^{\prime}$. Clearly the structure $\left(X_{\omega}, R_{\mathrm{i}}^{\prime} \cup R_{\mathrm{m}}^{\prime}\right)$, viewed as a graph, is connected. Therefore we can invoke König's Lemma to obtain an infinite sequence. 
If this sequence contains an infinite number of $R_{\mathrm{m}}^{\prime}$ transitions then there exists an infinite subsequence of the form

$$
x_{0} R_{\mathrm{m}}^{\prime} x_{1} R_{\mathrm{m}}^{\prime} x_{2} R_{\mathrm{m}}^{\prime} x_{3} \cdots
$$

By construction each of the $x_{i}$ is $R_{\mathrm{m}}$-maximal. But then transitivity of $R_{\mathrm{m}}$ implies that each of the $x_{i}$ belong to a different $\sim_{\varphi}$-equivalence class. A contradiction, since there are only finitely many such classes.

If the sequence obtained from König's Lemma has a finite number of $R_{\mathrm{m}}^{\prime}$-transitions, then there must be an infinite subsequence of the form $x_{0} R_{\mathrm{i}}^{\prime} x_{1} R_{\mathrm{i}}^{\prime} x_{2} R_{\mathrm{i}}^{\prime} x_{3} \cdots$ and a similar argument as above yields a contradiction. Thus no infinite sequence can exist, and therefore $X_{\omega}$ must be finite.

We could not simply invoke König's lemma to $X_{\omega}$ ordered by (restrictions of) $R_{\mathrm{i}}$ and $R_{\mathrm{m}}$ (like in [109]), because this could potentially yield an infinite chain whose states are not all $R_{\mathrm{i}}$-maximal or $R_{\mathrm{m}}$-maximal.

Finite submodel of $\mathfrak{F}$. Define the frame $\mathfrak{F}^{\prime}$ to be the subframe of $\kappa \mathfrak{F}$ generated by $X_{\omega}$, and $\mathfrak{M}^{\prime}$ as the model $\left(\mathfrak{F}^{\prime}, V^{\prime}\right)$, where $V^{\prime}(p)=V(p) \cap\left(X_{0} \cup X_{\omega}\right)$. Then by Lem. 5.3 we have

$$
\mathfrak{M}, y \Vdash \varphi \quad \text { iff } \quad \mathfrak{M}^{\prime}, y \Vdash \psi
$$

for all $y \in X_{\omega}$ and $\psi \in \operatorname{Subf}(\varphi)$. Therefore $\mathfrak{F}^{\prime}$ refutes $\varphi$. Since $\Theta$ is a canonical subframe logic and, $\mathfrak{F}^{\prime}$ is a subframe of $\kappa \mathfrak{F}$, we also have $\mathfrak{F}^{\prime} \Vdash \Theta$. This proves item 1 .

Proof of item 2. Assume the above construction started with a descriptive frame $\mathfrak{G}$ for $\Theta \oplus \Gamma$ that refutes $\varphi$. We have already seen that the resulting frame $\mathfrak{F}^{\prime}$ refutes $\varphi$ and validates $\Theta$, so it remains to show that $\mathfrak{F}^{\prime} \Vdash \Gamma$.

The assumption of strength, together with the construction of $X_{\omega}$, implies that each state $y \in X_{\omega}$ is $R_{\mathrm{m}}$-maximal. Therefore, since $R_{\mathrm{m}}$ is transitive, it follows from [109, Lem. 15] that $\mathfrak{G}^{\prime} \Vdash \Gamma$.

A.17 Theorem. Suppose $\Theta$ is a canonical extension of $\mathrm{S} 4 \otimes \mathrm{K} 4$ containing $\mathrm{BHL}$ that is closed under forming $R_{\mathrm{m}}$-cofinal subframes. Then:

1. $\Theta$ has the finite model property.

2. If $\Theta$ contains the strength axiom $\mathrm{S}_{\mathrm{a}}$, then for any $R_{\mathrm{m}}$-subframe logic $\Gamma \subseteq \mathcal{L}_{\mathrm{m}}$, the logic $\Theta \oplus \Gamma$ has the finite model property.

Proof of Theorem A.17. Let $\mathfrak{F}=\left(X, R_{\mathrm{i}}, R_{\mathrm{m}}, P\right)$ be a descriptive frame for $\Theta$ that refutes $\varphi$. Let $V$ be a valuations such that $(\mathfrak{F}, V) \Vdash \varphi$. In order to prove the theorem, we modify the proof of Thm. A.16 as follows: First, we modify the construction to obtain an $R_{\mathrm{m}}$-cofinal subframe. Second, we quotient out this new subframe to make it finite.

Let $\left\{C_{j} \mid j \in J\right\}$ be the set of all $R_{\mathrm{m}}$-final $R_{\mathrm{m}}$-clusters. Since $\mathfrak{F}$ is descriptive, every state $x$ has an $R_{\mathrm{m}}$ successor in an $R_{\mathrm{m}}$-final $R_{\mathrm{m}}$-cluster [23, Thm. 10.36]. Therefore, to achieve $R_{\mathrm{m}^{-}}$ cofinality, it suffices to add to $X_{\omega}$ a state from each $C_{j}$. In order to still be able to use Lem. 5.3 we add a (finite) set $F_{j}$ of states satisfying the precondition from Lem. 5.3 for each $j \in J$.

Constructing $F_{j}$. Let $C_{j}$ be a $R_{\mathrm{m}}$-final $R_{\mathrm{m}}$-cluster. Let $F_{j}^{\prime}$ be a minimal subset of $C_{j}$ such that for each $x \in C_{j}$ there is an $R_{\mathrm{m}}$-maximal $y \in F_{j, 0}$ such that $x \sim_{\varphi} y$. (We can find such $R_{\mathrm{m}}$-maximal states using [109, Lem. 14].) Since $C_{j}$ is a cluster and there are only finitely many $\sim_{\varphi}$-equivalence classes the set $F_{j}^{\prime}$ is finite. 
Now suppose $F_{j, k}$ has been defined. We give $F_{j, k+1}$. For each $y \in F_{j, k}$ let $Y_{y}$ be a minimal set of maximal $R_{\mathrm{i}}$-states $R_{\mathrm{i}}$-above $y$, such that for ever $z$ with $y R_{\mathrm{i}} z$ there exists $z^{\prime} \in Y_{y}$ such that $z \sim_{\varphi} z^{\prime}$. Define

$$
F_{j, k+1}=\bigcup_{y \in F_{j, k}} Y_{y} .
$$

We claim that this process is finite. If $z \in F_{j, k}$ is introduced in an earlier step, then $Y_{z}$ (used in the construction of $F_{j, k+1}$ ) is empty by minimality. If $z$ was introduced in the construction of $F_{j, k}$ as an element of some $Y_{y}$, where $y \in F_{j, k-1}$, then by construction and $R_{\mathrm{i}}$-maximality of the states in $F_{j, k-1}$ there are at most $c-k$ different $\sim_{\varphi}$-equivalence classes that $z$ can see. (Recall that $c$ denotes the number of $\sim_{\varphi}$-equivalence classes.) Therefore $\left|Y_{z}\right| \leq c-k$. This proves that the recursion terminates after $c$ steps.

Therefore, the set

$$
F_{j}=\bigcup_{0 \leq k \leq c} F_{j, k}
$$

is finite. In fact, each of the $F_{j}$ is bounded by $c^{c+1}$. Moreover, we claim that it satisfies the precondition from Lem. 5.3. Clearly, if $y \in F_{j}$ and $y R_{\mathrm{i}} z$, then by construction there exists $z^{\prime} \in F_{j}$ such that $z \sim_{\varphi} z^{\prime}$. If $y \in F_{j}$ and $y R_{\mathrm{m}} z$, then by (-3-p) we have $z \in C_{j}$. By construction there exists $z^{\prime} \in C_{j}$ such that $z \sim_{\varphi} z^{\prime}$ and by transitivity of $R_{\mathrm{m}}$ this implies $y R_{\mathrm{m}} z^{\prime}$.

An $R_{\mathrm{m}}$-cofinal subframe. Let $X_{\omega}$ be constructed as in the proof of Thm. A.16. Then by construction the subframe $\mathfrak{F}^{\dagger}$ of $\mathfrak{F}$ generated by

$$
\bar{X}_{\omega}=X_{\omega} \cup \bigcup\left\{F_{j} \mid j \in J\right\}
$$

is $R_{\mathrm{m}}$-cofinal. Let $V^{\dagger}$ be the induced valuation and set $\mathfrak{M}^{\dagger}=\left(\mathfrak{F}^{\dagger}, V^{\dagger}\right)$. Then since $\Theta$ is closed under $R_{\mathrm{m}}$-cofinal subframes we have $\mathfrak{F} \Vdash \Theta$, and as a consequence of Lem. 5.3 we have $\mathfrak{M}^{\dagger} \Downarrow \varphi$, so that $\mathfrak{F}^{\dagger} \| \varphi$.

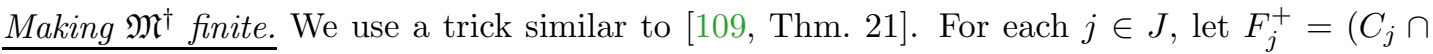
$\left.X_{\omega}\right) \cup F_{j}$. Since the $F_{j}$ and $X_{\omega}$ are uniformly bounded by $c^{c+1}+\left|X_{\omega}\right|$, so are the sets $F_{j}^{+}$ are uniformly bounded. Hence there are only finitely many non-isomorphic submodels of $\mathfrak{M}^{\dagger}$ generated by $F_{j}^{+}$. Identifying isomorphic such submodels yields a finite quotient of $\mathfrak{M}^{\dagger}$ that witnesses the claim.

Item 2. The second item is proved in a similar way as the second item of Thm. A.16. 